\title{
Die Gefässverbindungen der Placentakreisläufe eineiiger Zwillinge, ihre Entwicklung und ihre Folgen.
}

Von

\author{
Friedrich Schatz.
}

\author{
III. Die Acardii und ihre Verwandten. \\ (Schluss mit systematischem and alphabetischem Inhaltsverzeichniss der \\ ganzen Arbeit am Ende des Bandes.)
}

\section{Die anatomische Ausbildung der Acardii.}

Die anatomische Ausbildung der Acardii geht durchaus nicht ganz parallel der Aetiologie derselben und wird auch sonst besser and übersichtlicher in einem besonderen Capitel besprochen.

Bisherige Meinungen. Die naiven Ansichten bis zu Tiedemann, welche in der Einleitung aufgezählt sind, bedürfen einer Beleuchtung nicht.

H. Meckel, Dareste und Panum haben die abnormen Formen der Acardii zum grössten Theil für primäre Missbildungen und nur zum kleinsten Theil für die Folgen secundärer Ernährungsstörungen erklärt. In neuester Zeit hat Breus diese Ansicht weiter vertheidigt.

Claudius hat umgekehrt alle Acardii für primär normale Embryonen angesehen und alle abnormen Formen einheitlich aus den veränderten Circulationsverhältnissen der Acardii zu erkläreri gesucht.

Ahlfeld hat die Ansicht von Claudius beibehalten und nur noch bezüglich der Vollkommenheit der Formen auf die Wichtig- 
keit eines etwa vorhandenen, wenn auch nur rudimentären Herzens aufmerksam gemacht.

Kritik. Wie theilweise schon oben gezeigt ist und gleich noch woiter gezeigt werden soll, sind die Verhältnisse durchaus nicht so einfach, wie die genannten Autoren meinten. Diese haben durchweg ihre Beobachtungen, wenn sic auch an sich ganz richtig waren, zu sehr generalisirt und nicht genug bedacht, dass die Natur hier wic anderswo dieselben Resultate auf oft mehrfach, manchmal sogar vielfach verschiedenen Wegen erreiehen kann.

Die Vorstellung von Dareste und Panum ist uberhaupt nur auf die parasitären, aber nicht auf die freien, mit Nabelschnur versehenen Acardii anwendbar.

Primärer Herzmangel und zugleich Vorhandensein einer Nabelschnur - also eines Allantois-(Placenta-)Kreislaufes - an demselben Individuum ist unmöglich. Für die freien Acardii fehlt also bei Dareste und Panum dic Erklärung des Herzmangels ganz. Bei H. Meckel findet sich wenigstens primärer Herztod angedeutet.

Aber auch die übrigen Difformitäten der Acardii können nicht, wie diese Autoren wollen, als meist primär vorhanden ancrkannt, werden. Wären die Acardiiformen wirklich primär, so müssten sie mit den sonstigen Missbildungen wenigstens ungefähr parallel laufen. Das ist aber durchaus nicht der Fall. Wir finden diejenigen Missbildungen, welche sowohl bei den Acardiis als bei den Einlingen vorkommen, bei diesen in ganz anderem 7ahlenverhältnisse als bei den Acardiis, und viele Bildungen der Acardii werden bei Einlingen überhaupt nicht gefunden.

Missgebildete Zwillingsembryonen können zwar leichter Acardii werden als normale. Sie erfahren aber dann ebenso weitere und tiefgreifende Umgestaltungen, wie diejenigen Acardii erfahren, welche aus normalen Embryonen entstehen. Die Acardie erzengt also auch bei ihnen den Haupttheil der Missbildungen.

Jedenfalls sind unter allen Acardiis die aus abnormen Embryonen entstandenen in der Minderzahl. Dabei ist freilich unter "abnormem Embryo" nur die Gesammtgestaltung des Embryo zu verstehen. Deon kleinere Abnormitäten (Heteromorphie) finden sich, wie wir gesehen haben, umgekehrt am künftigen Acardius sehr häufig.

Auch die Vorstellung von Claudius kann nur auf den ersten Blick befriedigen. Man wird gern anerkennen, dass manche Or- 
gane nach Eintritt der Acardie durch ungenügende Ernährung atrophiren, andere ganz schwinden müssen. Da Claudius aber die Ernährung ausser von der Grösse der Bhtzufuhr nur von der Form des arteriellen Gefässsystems abhängig macht, so kommt man bei Prüfung seiner Hypothese an den einzelnen Fällen überaus häufig auf Widersprüche. Die Formen der Acardii entsprechen eben nur theilweise der Form des arteriellen Systems, um welches herum nach Claudius die Organe des Acardius erhalten bleiben sollen.

Die Wichtigkeit der Anwesenheit eines Herzens für die Vollkommenheit eines Acardius, welche Ahlfeld hervorhebt, ist durchaus nicht zu verkennen. Sie trifft aber auch nur bei einigen Fällen zu. Manche sehr vollkommene Acardii haben kein Herz, und manche, welche ein Herz haben, sind trotzdem recht unvollkommen.

Eigene Resultate. Ich selbst bin bei meinen Untersuchungen bezüglich der anatomischen Ausbildung der Acardii zu folgenden Ergebnissen gekommen.

Im Allgemeinen muss die Form und Organisation derjenigen Embryonen, aus welchen später Acardii werden, als die gewöhnliche und normale angesehen werden. Im Allgemeinen entstehen also die Acardii aus normalen Embryonen.

Wir kennen schon eine Anzahl von Acardii (Breus, Ahlfeld, Metzner, Koch), denen trotz der Acardie eigentlich nichts weiter fehlt, als das Herz. Da bei Entwickelung des Allantoiskreislaufes auch dieses dagewesen sein muss, so hat ihnen damals offenbar gar nichts gefehlt.

Bei anderen Fällen findet man zwar ausser dem Herzen auch den Kopf nicht mehr, dafür aber wenigstens einen Stumpf davon (Fall von Hempel), welcher beweist, dass der Kopf früher vorhanden war.

Aehnlich kann man bei sehr vielen Acardiis auch den Defect anderer Organe als secundär geradezu nachweisen. Von dem Acardius von Nicot le Cat (Tiedemann, S. 33), welcher 12"6"“ lang war, wird berichtet: "An dem oberen Theil des Körpers nahm man deutliche Spuren einer gewaltsamen Zerstörung des Kopfes, des Halses und der oberen Extremitäten wahr." Bei dem Fall von Kalten,bach fanden sich lose Knochenstïcke im Eisack des Acardius: offenbar Reste von abgefallenen Körpertheilen. Bei dem Acardius acephalus von Eckardt (Centralbl. f. Gynäk., 1889, S. 547) wurden die Untersehenkelknochen frei neben dem Hals 
des gesunden Kindes geboren, und der Autor fand an den Beinen des Acephalus keine entsprechenden Oeffnungen oder narbigen Einziehungen, ein Beweis, wie gering die Narben sein können, welche der nekrotische Abfall selbst grosser Körpertheile hinterlässt. Die (scheinbare) Abwesenheit soleher Narben kann also niemals beweisen, dass die betreffenden Körpertheile schon primär gefehlt haben.

Bei manchen Fällen ist das Fehlen auch von inneren Organen, 2. B. von Muskeln ete., als secundärer Verlust durch Verfettung derselben etc. (Fall Eysell) nachweisbar.

Alle solche Verluste von Körpertheilen weisen darauf hin, dass der Körper vor Eintritt der Acardie vollständiger gewesen ist. Sie geben aber keinen Beweis dafür, dass er wirklich vollständig war. Wenn ein Acardius auch mehrere Glieder verlieren kann und verliert, so braucht er deshalb doch nicht primär vollständig oder gar normal gewesen zu sein. Das wollen wir aber wissen: und man muss und kann die Verhältnisse viel eingehender und klarer behandeln.

Zunächst sind durchaus zu unterscheiden

1. die primären Abnormitäten und Defeete der Acardii, welche schon vor, und 2. die secundären, welche erst nach Eintritt der Acardie vorhanden sind.

Die primären Abnormitäten und Defecte der Acardii.

Sie zerfallen sich wieder in: a) wesentliche und b) zufällige.

b) Zufällige primäre sind solche, welche auch bei Einlingen vorkommen, und welche mit der Acardie weder als Ursachen noch als Folgen etwas zu thun haben. z. B. Hemicephalie, Spina bifida ete. - siehe oben "Falsche Fälle".

Es ist nicht zu verkennen, dass Zwillinge, welche überhaupt mit beliebigen Missbildungen behaftet sind, leichter auch solche Abnormitäten haben werden, welche zu Acardie führen können. Trotzdem aber sind jene Missbildungen als zufällige anzusehen. Sie kommen hier nicht weiter in Betracht und bleiben deshalb unerörtert.

a) Wesentliche primäre Abnormitäten und Defecte der Acardii sind solche, welche, sei es als Ursachen, sei es als Folgen, zur Acardie gehören.

Nun brauchen aber die Ursachen der Acardie gar nicht im Körper des Zwillings, sondern können anch ausserhalb desselben 
- in der Nabelschnur, in der Placenta - liegen. In diesen Fällen sind die zugehörigen späteren Acardii principiell als ursprünglich in jeder Richtung normale (orthomorphe) Früchte anzusehen. Es sind die Acardii der Gruppen I, V, VIII, IX. Sie sind aber von allen Acardiis offenbar die weniger zahlreichen.

Alle übrigen Acardii, welche also die Ursachen der Acardie in ihrem Körper haben und welche die grössere Hälfte aller Acardii ausmachen, können als ursprünglich völlig normal nicht angesehen werden. Allerdings sind auch sie zumeist primär vollständig, haben also keine Defecte. Sie haben aber Abnormitäten im Verlauf ihrer Nabelvene und in deren Umgebung, welche das Stromhinderniss und damit die Acardie erzeugen. Diese Abnormitäten, welche ich zusammen als Heteromorphie bezeichnet und oben, Bd. 58 , S. 6 , schon charakterisirt habe, werden uns freilich, wie dort schon gesagt ist, erst dann genügend vollständig und durchsichtig bekannt werden, wenn wir eine grössere Zahl heteromorpher Zwillinge werden kennen gelernt haben aus den Arten, welche nicht zu Acardiis geworden und dadurch weiter zerstört sind, d. s. heteromorphe Mikrocardii (Gruppe III, Bd. 58, S. 1) und heteromorphe Makrocardii (Gruppe IV, Bd. 58, S. 28).

Nach den bisherigen Beobachtungen gehört zu ihnen und damit zu den wesentlichen primären Abnormitäten und Defecten der betreffenden Acardii:

a) Situs transversus (s. Bd. XXIX, S. 424).

Er ist an sich bei eineiigen Zwilligen häufiger als sonst. Ist er ganz vollkommen, so wird er freilich kaum zu Stromhindernissen in der Nabelvene führen, dies jedoch um so mehr, wenn er unvollkommen ist.

Vollkommener oder unvollkommener Situs transversus bei Acardie und Hemiacardie findet sich z. B. in den Fällen von:

Mery (bei Tiedemann, S. 70) Aorta rechts, Hohlader links;

Lang (bei Vrolik, Tab. 50);

Sulzmann (bei Tiedemann, S. 32);

Klein (bei Tiedemann, S. 35), Aorta links von der Wirbelsäule, doch rechts von der Vena cava;

Barkow (s. oben Bd. 58, S. 9).

Wiewohl dieser Fall nur einen heteromorphen Mikrocardius (Pseudohemiacardius) darstellt, muss er doch hier mit genannt werden, weil das Nichteintreten der Acardie nur in der Placenta begründet war: durch 
Fehlen beider Anastomosen auf der Placenta. Wären diese rorbanden gewesen, so wäre wirkliche Acardie eingetreten. and 2).

Koch I. (dieses Archiv, XXVI, S. 290, u. Tafel III, Figur 1

Koch II. (dieses Archiv, Bd. XXVI, S. 307, Taf. III, Fig. 6).

Zweifelhaft ist der Situs transversus in den Fällen von:

Roederer (s. oben Bd. 60, S. 139),

C. Mayer (Verhandl. d. Gesellsch. f. Gebh. ete., Berlin 1846, S. 128 ,

Vrolik (Vrolik, Taf. L); Vrolik fasst die Vend cava als links von der Aorta liegend auf.

Wie der Situs transversus etwa wirkt. ist recht deutlich zu ersehen bei dem. I. Fall Koch's. Aorta liegt rechts, Vena cava links. Das Herz, von dem keine Spur mehr zu finden war, das aber schon wegen des Vorhandenseins einer $23 \mathrm{~cm}$ langen, mit zwei Arterien versehenen Nabelschnur fruher dagewesen sein muss, wird rechts gelegen haben. Rechts ist aber auch die (kleine) Leber. Hier hat eine regelrechte Bildung der Nabelvene in der Lebergegend nicht stattfinden können. Es erweiterte sich zwar die linke Burow'sche Vene als Ersatzmabelvene. Die Speisung des Herzens mit Blut von der Placenta her blieb dabei aber gegenüber derjenigen des Herzens des gesunden $Z$ willings doch im Nachtheil. Das dadurch geschwächte Herz konnte dem grösseren arteriellen Druck des gesunden um so weniger Stand halten, als die arterielle Placentanostomose sich schon zwischen den Nabelschnurarterien an der Insertion der beiden Nabelschnüre befand.

Bei der Betrachtung des Einflusses des Situs transversus aut die Aetiologie der Acardie ist aber doch auch wieder hervorzuheben, dass in der Gesammtzahl der Acardii immer nur ein kleiner Theil Situs transversus zeigt. Die bei weitem meisten Acardii müssen also auch in dieser Hinsicht primär normal gewesen sein. S. 53).

b) Nichtentwicklung des Ductus venosus Arantii (s. Bd. 58,

c) Störung in der Entwicklung der Leber, deren Gefässe eine Zeit lang allein die Fortsetzung der Lebervene bilden (s. Bd. 58, S. 51 u. 52).

d) Störungen in der Entwicklung der Nabelvene (s. Bd. 58, S. $49 \mathrm{ff}$.).

e) Stenose der sehon entwickelten Nabelvene.

f) Nabelstenose mit folgender Stenose der Nabelvene (s. Bd. 58, S. 97 ).

g) Nabelschnurbruch mit folgender Stenose oder Abknickung der Nabelvene (s. Bd. 58, S. 72 ff.). 
Um diese Abnormitäten gruppiren sich dann noch eine Anzahl weiterer - theils als unmittelbare Ursachen, theils als unmittelbare Folgen der ersteren, so: Verkümmerung der Leber, Narben im Peritoncum, Versehluss der Vena cava infer. etc. Auch sie müssen als zur Heteromorphie gehörig und somit der Acardic gegenüber als primäre wesentliche Abnormitäten angesehen werden.--

Viel auffälliger als diese primären treten hervor:

Die secundären Abnormitäten und Defecte der Acardi.

Um ihre Erklärung hat sich denn auch so gut wie allein die umfangreiche literarische Discussion gedreht, um so mehr, als man die unscheinbaren primären noch nicht kannte.

Auch ich glaube, dass man alle Hauptveränderungen, welche nach Eintritt der Acardie stattfinden, lediglich dureh die grössere oder geringere Vollkommenheit erklären kann, mit welcher die Blutcirculation in den einzelnen Theilen des Acardius vom fremden Herzen her unterhalten wird. Aber die Vorgänge sind nicht entfernt so einfach, wie sie sich Claudius vorstellte.

Wir betrachten sie am Uebersichtlichsten unter folgenden 3 Gesichtspunkten, welche sich auf die Blutcirculation beziehen:

1. Genügender Zufluss und genügender Abfluss zu und ron dem Acardius;

2. Ungenügender Zufluss und genügender Abfluss zu und von dem Acardius;

3. Genügender Zufluss und ungenïgender Abfluss zu und von dem Acardius.

Erzeugt werden diese drei Combinationen hauptsächlich durch die Beschaffenheit der Anastomosen auf der Placenta, welche den nunmehrigen Acardius ernähren müssen. Diese Anastomosen zeigen, wie ich in den früheren Abschnitten (Bd. XXIV, S. 356-358, und Bd. XXVII, S. 61-63) dargelegt habe und wie auf den Placenten mit dem Typus D, Bd. XXIV, Taf. I, $D_{1}$, Taf. V, $D_{2}, D_{3}$, $\mathrm{D}_{4}, \mathrm{D}_{8}, \mathrm{~B} d$. XXVII, Taf. I, $\mathrm{D}_{9}$, Taf. II, $\mathrm{D}_{10}$, ohne Weiteres ersicht lich ist, alle möglichen gegenseitigen Verhältnisse, und zwar sowohl je die arteriellen und die venösen unter sich, als die je zusammengehörigen arteriellen und venösen gegeneinander.

Wenn die Anastomosen aus Placentaarterien und -Venen erster Ordnung oder gar aus Nabelschnurarterie und -Vene entspringen resp. dort münden, so haben sie im Allgemeinen das grösste Lumen 
und gewähren dadurch dem Acardius volle Ernährung. Je weiter rückwärts - also von Placentaarterien resp. Venen höherer Ordnung sie entspringen resp. münden, um so kleiner ist im Allgemeinen ihr Lumen und um so geringer damit die Ernährung des Acardius durch sie.

Weiter ist gewöhnlich das Lumen der venösen Anastomose dünner als das der arteriellen. Da schon ein gleiches Lumen der venösen Anastomose als relativ ungenügend angesehen werden muss, weil es Stauung erzengt, so ist dies bei geringerem Lumen umsomehr der Fall. Doch finden sich, wie in allen diesen Verhältnissen, so auch hier, nicht selten Ausnahmen, sodass eine venöse Anastomose (besonders gegenüber einer’ engen arteriellen) recht wohl das eine oder andere Mal genügen kann.

Unter meinen Placenten der Tafeln I, V, VI, VII ist das Calliber der venösen Anastomose enger als das der arteriellen bei $D_{2}$ (Taf. V), $D_{10}$ (Taf. VII), etwa gleich bei $D_{1}$ (Tafel I), $D_{4}$ (Tafel V), grösser bei $\mathrm{D}_{3} \mathrm{D}_{8}\left(\right.$ Tafel V), $\mathrm{D}_{9}$ (Tafel VI).

Freilich kommt auf Seite des venösen Rückflusses vom Acardius zur Placenta meist auch noch das Stromhinderniss hinzu, welches die Acardie veranlasst hat. Dieses wird den Blutabfluss aus dem Acardius um so ungenügender machen, wenn sich um dasselbe herum genügende Ersatzbahnen nicht entwickelt haben.

Beide engen Stellen zusammen - die venösen Anastomosen auf der Placenta und das Stromhinderniss, welches die Acardie veranlasst - hat schaffen für den Acardius so häufig eine absolute oder mindestens relative Insufficienz des venösen Abflusses, dass die zugehörigen Formen der Acardii bei weitem die häufigsten sind, und dass auch bei den übrigen Acardiis Stauungserscheinungen so gut wie nie fehlen.

Die Einwirkungen der genannten drei Combinationen auf die anatomische Ausbildung der Acardii sind folgende:

ad 1. Die Einwirkung von genügendem Blutzufluss und genügendem Blutabfluss zu und von dem Acardius auf dessen anatomische Ausbildung.

Im Allgemeinen bedarf der Acardius, auch wenn er recht vollständig ausgebildet wird, nicht des normalgrossen Blutstromes eines gewöhnlichen Fötus, sondern eines relativ nur geringen und dies wahrscheinlich deshalb, weil das Blut in ihm wegen grosser Entfernung vom bewegenden Herzen nur langsam fliesst. Man muss 
auch dementsprechend taxiren. Z. B. bei dem recht gut ausgebildeten Fall von Breus (dies. Arch., Bd. 58, Taf. IV, Fig. 41) ist sowohl die zuführende einzige Nabelschnurarterie, als auch die abführende Nabelschnurvene ziemlich eng. Ebenso bei dem sehr vollkommen ausgebildeten Acardius Koch II (dies. Arch., Bd. XXVI, Taf. III, Fig. 5). Wesentlich ist, dass bei diesen beiden Fällen die Nabelschnurarterie des Acardius mit der Nabelschnurarterie des gesunden Zwillings direct anastomosirte, wodurch der Blutdruck möglichst hoch blieb. Bei den übrigen gut ausgebildeten Acardiis der Literatur ist die Placenta gewöhnlich nicht bekannt und so eine Controle nicht möglich. Bei dem Fall von Hempel (De monstris acephalis, Diss., Hafniae 1850) ist die Placenta auch gut abgebildet; und Art. wie Ven. der Nabelschnur des Acardius zweigen eben so günstig ron denjenigen des normalen Mitzwillings an der Insertion in die Placenta ab, wie bei jenen Fällen. Trotzdem ist der Acardius nicht ganz vollständig. Der Kopf ist deutlich abgefallen. Einer gewissen Circulationsgrösse bedarf es also zu möglichst vollkommener Weiterbildung des Acardius doch. Ist diese Grösse aber vorhanden, so ist es für die äussere Vollkommenheit des Acardius ohne wesentlichen Einfluss, wo am Acardius der arterielle Zufluss and wo der venöse Abfluss statthat. Es wird eben der ganze Körper unter Circulation gehalten und weiter entwickelt, oder es entstehen höchstens nebensächliche Defecte.

Anders wenn das Blut, welches durch die Nabelschnurarterien des herztodten Zwillings in dessen Aortensystem eintritt, zu wenig ist, um das ganze Arteriensystem unter genügendem Blutdruck zu erhalten, besonders dann, wenn zugleich der Blutabfluss genügend weit ist, sodass die Stauung nicht gross genug wird, um dadurch die Füllung des Gefässsystems wieder etwas zu erhöhen - wenn also ungenügender Blutzufluss mit genügendem Blutabfluss zusammentrifft.

ad. 2. Die Einwirkung von ungenügendem Blutzufluss und genügendem Blutabfluss zu und ron dem Acardius auf dessen anatomische Ausbildung.

Da können allerdings im Allgemeinen nur die Blutbahnen mit kürzeren Gefässschlingen unter Circulation bleiben, während das Blut in den längeren Gefässschlingen stagniren und diese Blutbahnen veröden müssen.

Der Vorgang ist aber doch durchaus nicht so einfach, wie Claudius ihn sich vorgestellt und andere gelehrt hat. Es kommt 
bei der Frage, welche Gefässschlingen in Circulation und welche Theile des Arcardius dadurch erhalten bleiben, durchaus nicht nur auf die Länge oder Kürze und auf das Kaliber der Gefässschlingen an, also nicht, wie Claudius es darstellt, nur darauf, wieviel dem Acardius und seinen einzelnen Theilen durch die Nabelschnurarterien Blut zugeführt wird, sondern in noch viel höherem llaasse darauf, wie lang und weit der venöse Theil der Gefässschlingen ist; also auf welehen Wegen und wie bequem das Blut wieder zurück zur Placenta gelangen kann.

Die arterielle Zufuhr geschieht bei aller Acardis mit ganz seltenen Ausnahmen z. B. bei dem Fall Sehatz II (S. oben Bd. 58 S. 74), bei Fall Spliedt (Bd. 60, S. 99), vielleicht anch bei Fall Moldenhauer (Bd. 58, S. 66) durch eine oder beide Art. iliac. Nach Claudius müssten also die Acardii so gut wie immer Acephali sein. Sie müssten immer gewissermaassen die Form des arteriellen Gefässsystems umkleiden, und ihre Organe und Formen müssten sich immer und nur je nach der Grösse des Blutzuflusses mehr weniger weit und vollkommen an die Beckenpartie, wo der Zufluss geschieht, anschliessen. Claudius hat dies ja auch bis zu einem gewissen Grade (bei den Accphalis) bestätigt gefunden, und sich damit zufrieden gegeben. Bei genauerer Durchsicht der Fälle findet man aber so viel Mannigfaltigkeiten und Abweichungen, dass man das von Claudius angenommene gar zu einfache Eintheilungsprincip nach der Grösse des Blutzuflusses ron den Nabelarterien her aufgeben muss.

Noch viel weniger kommt man zu Stande, wenn man wie Claudius und Sippel (1. c. S. 5) sogar die Richtung des Blutstromes resp. den spitzwinkeligen Abgang der Arterien für das Fehlen der zugehörigen Organe verantwortlich machen will.

Nur wenn man den Ort des venösen Abflusses zur hauptsächlichen Grundlage der Betrachtungen nimmt, kommt man unter Berücksichtigung der oben dargelegten Aetiologie der Acardii zu einem befriedigenden System für die Morphologie der Acardii.

Es kann das auch nicht anderes sein. Nicht der Zutritt von Blut zu einem Organe ist es, welcher dasselbe ernährt, sondern die Circulation des Blutes in demselben, und diese ist trotz genügenden Zuflusses nur gesichert bei genügend freiem Abfluss durch die Venen. Ohne letzteren tritt Gangrän ein. 
Non haben wir aber gesehen, dass, so constant bei den Acardiis die Stelle für den arteriellen Zufluss ist, so ineonstant und wechselnd die Stelle für den venösen Abfluss. Wir finden die abführende Vene bei den Acardiis nicht nur im Becken in der Nähe der zuführenden Arterien, (Burow'sche Venen), sondern auch in der Mitte (Nabelvene), odér sogar an der oberen Hälfte des Rumpfes (Dottervene, Jugularvene, Bauchdeckenvene) mauchmal auch an zwei von einander entlegenen Stellen. Diese grosse Nannigfaltigkeit des Ortes des venösen Abflusses erklärt die durchaus verschiedene Ernährung und den wechselnden Fortbestand der einzelnen Theile der Acardii. Im Uebrigen ist es dann ziemlich gleichgültig, ob der venöse Abfluss durch die Allantois-(Nabel-)vene oder durch ihre Ersatzvenen oder durch die Dottervene oder durch beiderlei besorgt wird.

Es wird zweckmässig sein, wenn auch nur in grossen Zügen und in Form eines Schemas an den Formengruppen der Acardii den grossen

Einfluss, welchen der Ort des venösen Abflusses ausübt, zu erläutern. Es gelingt dies in Allgemeinen sehr wohl, wenn man sich nur nicht darauf steift, jetzt schon glejch auch für die Einzelheiten Erklärungen zu suchen.

Am durchsichtigsten und am beweisendsten gestalten sich die Verhältnisse bei den Acormis. Wie bei allen Acardiis, mit ganz seltenen Ausnahmen, so ist auch bei ibnen der arterielle Zufluss zu allen Zeiten für die untere Körperhälfte günstiger, als für die obere. Dort münden die Nabelarterien. Da bei ihnen aber die Allantoisvene vollständig verödet, und damit gewöhnlich auch die untere Hohlvene gar nicht oder nicht genügend zur Entwickelung kommt, so fehlt für die untere Körperhälfte der venöse Abfluss oder er ist wenigstens viel schwieriger; denn die als Collaterale der ganzen Allantoisvene erhaltene gemeinschaftliche Dottervene führt nur das Blut der oberen Hohlvene bequem ab. Dadurch bleibt die obere. Körperhälfte allein unter dauernder Circulation, und entwickelt sich allein weiter. Dass dabei der Kopf den Löwenantheil erhält, und fast. allein ansgebildet wird, während die oberen Extremitäten kaum angedeutet sind, kann nicht Wunder nehmen, weil zu dieser Zeit des intrauterinen Lebens der Kopf allein die obere Körperhälfte darstellt - das Herz liegt zu dieser 
Zeit im Hals - und die oberen Extremitäten noch kaum angelegt sind (s. den Acormus von Barkow (Bd. 58, Taf. III, Fig. 37). Aehnlich wie bei der Dottervene ist das Verhältniss, wenn die Allantoisvene zwar erhalten, jedoch die untere Hohlvene obliterirt ist. Es bleibt dann der Kopf und der obere Theil des Rumpfes unter Circulation und bestehen, aber die untere Körperhälfte fehlt.

Anders wird das Verhältniss schon, wenn neben der bei Bestand gebliebenen gemeinsamen Dottervene die Allantois(Nabel-) vene in der Jebergegend nicht ganz obliterirt, oder wenn sie durch eine ron unten kommende Collateralvene ersetzt wird, so dass der Acardius zwei Nabelschnurvenen besitzt. Dann besorgt die Dottervene den Rückfluss für die obere und die Nabelvene den Rückfluss für die untere Körperhälfte, und es können beide Körperhälften ziemlich rollkommene Entwicklung zeigen (s. d. Fall von Poppel, Bd. 60, Taf. IX, Fig. 67, 68).

Aehnlick ist der Erfolg, wenn die in der Lebergegend obliterirte Nabelvene zugleich durch eine nach der oberen und eine nach unteren Körperhälfte gehende Collateralvene ersetzt wird. Es bleibt dann nahezu der ganze Körper unter Circulation (s. d. Fall von Brandau Fig. 59, 60, Bd. 60, Taf. VII). Er ist zwar Hemiacardius, würde aber als Holoacardius nicht viel anders aussehen (s. auch Fall Sowoidnich Bd. 58, Taf. VI, Fig. 46).

l'ehlt die gemeinsame Dottervene, entweder weil sie bei Eintritt der Acardie schon obliterirt war oder weil (bei eineiigen Zwillingen mit 2 Dottern) ein gemeinsehaftlicher Dotterkreislauf überhaupt nicht bestanden hat, und führt dann die Nabelvene das Blut durch eine oder beide Venae collaterales umbilico-iliacae, (Burow'schen Venen) nur aus der unteren Körperhälfte bequem zurück, so entwickelt sich nur oder bevorzugt die untere Körperhälfte, wie wir es bei der so grossen Anzahl der gewöhnliehen A cephali finden. Sie wird im schlimmsten Falle nur das Beckenstück eines Embryos mit oder ohne untere Extremitäten.

Besteht endlich bei Eintritt der Acardie die Nabelvene in der Lebergegend unbehindert fort, so dass sie den Rückfluss des Blutes von der Mitte des Körpers aus besorgt, so müssten die Ernährungsstörungen eigentlich überall ziemlich gleich sein und es entstehen dadurch auch wirklich öfter Cormi (Amorphi in der Bedeutung, dass sie nur oder hauptsächlich den Rumpf zur Entwickelung bringen).

Aber in solchem Falle, wo die Abflussverhältnisse im Venen- 
system für den ganzen Körper ziemlich oder vollkommen gleichmässig sind, da erkennt man denn doch, dass auch der Ort der arteriellen Zufuhr nicht ganz ohne Einfluss bleibt, besonders nicht bei denjenigen Acardiis, welche durch primären Herztod entstehen. Dann ist wirklich der in der unteren Körperhälfte geschehende arterielle Zufluss die Ursache, dass die untere Körperhälfte eine etwas bessere Ernährung erfährt, als die obere, wie es Claudius für alle Fälle gelten lassen wollte. Der Kopf stirbt dann leicht ganz $a b$ und fällt $a b$, wie bei dem Fall von Sippel, nicht selten auch ein Theil des Thorax, wie bei dem Falle von Eysell (Bd. 55, Taf. XXII). Freilich fällt dann gewöhnlich auch noch ein Theil der unteren Körperhälfte mitab (eine untere Extremität, einzelne Zehen etc.).

Dieses Absterben und event. Abfallen muss man sich so erklären, dass das Blut in den zu langen Gefässschlingen wegen seines zu langsamen Laufes zur Gerinnung kommt, woraus Absterben der durch sie ernährten Theile oder, bei nicht allseitiger Gerinnung, wenigstens Atrophie derselben resultirt. -

Es entwickelt sich also bei zu geringer arterieller Zufuhr immer nur ein Theil des Acardius weiter. Dass es stets zunächst derjenige ist, welcher um die Stelle des venösen Abflusses herumliegt, hat seinen Grund darin, dass das Venensystem mit seinem geringeren Blutdruck gegen jede Störung weit empfindlicher ist als das Arteriensystem. Längere venöse Blutläufe werden sogar auch relativ leichter gestört als kürzere. Von gleichlangen Gefässschlingen werden solche mit grösserer arterieller Hälfte (bis zu den Capillaren) stets das Uebergewicht gewinnen vor solchen mit grösserer venöser Hälfte. Das Venensystem bleibt also gegenüber dem Arteriensystem bei der Frage, welche Theile des Arcadius bei Bestand bleiben werden, immer das maassgebendere.

Es darf nicht verwundern, dass die durch Abfallen selbst grosser Theile entstehenden Narben am wachsenden Embryo und bei Fehlen jeder Eiterung oft so zart und klein werden, dass man sie kaum oder gar nicht erkennt. Besonders oft wird angenommen, dass der Kopf gar nicht zur Entwickelung gekommen sei und dass ein vorhandener kleiner Haarbüschel die Stelle des atrophirten Kopfes bedeute. Auf mich macht dieser Haarbüschel den Eindruck, dass er nur der kleine Rest der Kopfhaut ist, welcher beim Abfallen des macerirten Kopfes am Nacken zurück- und lebend blieb, und dann in die Defektwunde anf den Stumpf 
so einheilte, dass dadurch die Narbe so gut wie nieht zu sehen ist. Das eingeheilte Stück Kopfhaut entwickelt natürlich die ihm zukommenden Kopfhaare, kann aber nicht das ganze Haupthaar darstellen. Aehnlich ist es bei anderen Stümpfen z. B. der Extremitäten. Der nach dem Abfallen eines Fusses weiter wachsende Stumpf setzt in der gewöhnlich noch langen Zeit des intrauterinen Luebens neben und über die freigelegten Knochen etc. wieder reichlich soricl Waterial ab, dass der oft relativ colossale Amputationsstumpf noch sehr sehön bedeckt erseheint. Das so oft eintretende Oedem unterstützt den Vorgang (s. auch S. 203).

ad 3. Die Einwirkung ron genügendem Blutzufluss und ungenügendem Blutabfluss zu und von dem Areardius auf dessen anatomische Ausbildung.

Wie uns Physiologie and Pathologie täglich lehren, ist im ganzen Körper der renöse Rückfluss wegen seines geringeren Druckes überall viel empfindlicher gegen Störungen, als der arterielle Zufluss. Die Natur hat dem entsprechend das Kaliber der Venen, mögen sie einfach oder doppelt sein, im Allgemeinen immer mindestens von doppelt grossem Querschnitt construirt. Schon bei gleich grossem Quersehnitt von zusammengehöriger Arterie und Vene tritt Stauung und Oedem ein. Es muss also auch beim Acardius bei gleichem Kaliber der abführenden Vene und der zuführenden Arterie Oedem und Ernährungsstörung, bei geringerem Kaliber der Vene aber ausserdem leicht noeh Gangrän eintreten.

Dic Verengung oder Behinderung in der Nabelschnurvene braucht nur gering, oder kaum merkbar zu sein, un doch schon starkes Oedem zu crzengen, sobald nur der arterielle Zufluss zum Acardius gross ist.

Bei dem Acardius von A. Martin (Berlinische Zeitschr. für Geburtshülfe und Gynaekologie, 1877, S. 45) betrug die Oedemflüssigkeit allein $2000 \mathrm{~g}$, während der ganze Acardius $3600 \mathrm{~g}$ wog. Der Blutzufluss zum Acardius geschah durch zwei Nabelschnurarterien, war also reichlich. Die Nabelschnur-Vene vereinigte sich noch in der Schnur mit derjenigen des normalen Zwillings. Eine deutliche Verengung der Nabelschnurrene des Acardius liess sich nicht konstatiren, sie war also vielleicht nur relatir. Es scheint zur Erzeugung der Stauung und des grossen Oedems schon der Umstand genügt zu haben, dass das vom Acardius durch dessen 
Nabelschnurvene zur Placenta zurückkehrende Blut direct in die Nabelschnurvene des gesunden Zwillings fliessen musste, deren Blut in entgegengesetzter Richtung ankam. Es ist nicht angegeben, ob an der Verbindungsstelle technisch correcte Vorrichtungen zur möglichsten Vermeidung der Stauung vorhanden waren. Vielleicht genügte zur Erzeugung der Stauung im Acardius sogar schon die Druckdifferenz zwischen beiderlei Blut an der Vereinigung beider Nabelschnurvenen. Solche musste vorhanden sein, weil das Blut, welches vom gesunden Zwilling her durch die Placenta geflossen war, einen viel kürzeren Weg zurückgelegt hatte, als das, welches durch den Acardius gegangen war.

Allerdings hat der gehemmte venöse Abfluss im Allgemeinen auch einen gewissen günstigen Einfluss auf die Entwicklung des Acardius wenigstens in jenen Fällen, in welchen vermöge des ungenügenden arteriellen Zuflusses nur ein Theil des Körpers weiter ernährt wird - nämlich dadurch, dass der gehemmte venöse Abfluss das ganze Gefässsystem des Acardius unter grösserem Blutdruck und damit auch mehr Gefässe unter Circulation hält, als es bei freierem venösen Abfluss der Fall sein würde. Der A cardius wird als o, resp. bleibt dadurch etwas vollkommener. Doch wird dieser Vortheil weit überholt dureh den Nachtheil, welchen das gleichzeitig eintretende starke Oedem erzengt. Bei allen Fällen mit genügendem arteriellen Zufluss und entsprechender vollkommener Entwicklung des Acardius kommt nur der Nachtheil des Oedems zur Geltung. Aber auch bei den Fällen mit ungenügendem arteriellen Zufluss erdrückt das Oedem leicht ebensoviel oder noch mehr als was die grössere Füllung der Gefässe mehr erzeugt oder erhalten hat, wenn auch oft an anderen Stellen. Es b]eibt also schliesslich für alle Fälle eine mehr weniger starke Ernährungsstörung durch das Oedem und in manchen Fällen auch eine ganz unmenschliche äussere Form übrig.

Besonders gross wird die Ernährungsstörung und Formveränderung des Acardius, wenn der Weg für den venösen Blut-Abflusss recht deutlich und stark enger ist als der für den artiellen Blutzufluss. Dies ist, wie oben gezeigt, leicht und hänfig der Fall an zwei Stellen, nämlich einmal bei dem Stromhinderniss, welches zur Entstehung der Acardie geführt hat und damn an der venösen Anastomose auf der Placenta — an jener Stelle besonders, wenn dieselbe nịcht durch erweiterte Ersatzvenen überbrïckt oder umgangen werden konnte, also besonders bei den Verengungen der 
Nabelschnurvene und an der venösen Placentaanastomose besonders, wenn diese, wie so leicht und häufig von einer Placentavene höherer Ordnung ab- resp. zu einer solchen hingeht.

Es ist nicht nöthig alle verschiedenen Grade der Enge dieser Stellen und ihre Folgen durchzusprechen. Es genügt zunächst nur die. höchsten Grade zu betrachten, welche zur Bildung der Amorphi führen.

Diejenigen Amorphi, welche durch Verengung der Nabelschnurvene entstehen, habe ich oben als "typisehe Amorphi globuli" bezeichnet. Bei ihnen ist diese Verengung zugleich sowohl die Ursache der Acardie als auch die Ursache der Amorphie, indem sie zuerst den Blutstrom von der Placenta zum Herzen vermindert und dadurch Herztod bewirkt und dann nach diesem bei umgekehrtem Blutlauf den Abfluss des Blutes vom Acardius nach der Placenta hin beeinträchtigt und dadurch Stauung, Oedem und vielfach Gangrän erzeugt.

Bei den anderen (nicht typischen) Amorphis ist die enge Stelle, welche die Acardie bewirkt, von derjenigen, welche die Amorphie erzeugt, verschieden. Die erste ist entweder von Anfang an nicht so eng oder ist durch genügend erweiterte Collateralvenen so ersetzt, dass durch sie allein der Rückfluss des Blutes vom Acardius nicht bis zur Entstehung von Amorphie gehindert würde. Dies geschieht erst durch Hinzutritt der starken Enge der venösen Placentaanastomose oder auch einer andern resp. dritten Stelle des venösen Weges zwischen Placenta und Acardius.

Die Folgen sind beide Male (bei den typischen und bei den nicht typischen Amorphis) nahezu dieselben. Tritt die Acardie schon in der Zeit ein, wo die Extremitäten noch nicht oder wenig entwickelt sind, so bewirkt das eintretende starke Oedem eine sackartige Ausweitung der ganzen Körperhaut, an welcher die Reste von Extremitäten manchmal kaum erkennbar sind und in welche manchmal sogar der Schädet mit eingeschlossen wird. Andere Male wird der ganze Kopf gangränös und fällt ebenso ab, wie die etwa schon stärker vorstehenden Extremitäten. Im Hautsack selbst werden die (weichen) Eingeweide am frühesten und am meisten zerstort und event. resorbirt. Wir sehen ja, dass diese sehon bei der Mikrocardiis am frühesten und meisten leiden und einschmelzen (S. Bd. 55, S. 614).

Welche Theile rom (resistenteren) Knochen- und Huskel- 
system erhalten bleiben oder gar etwas weiter gebildet werden, ist im Einzelfall kaum mit Sicherheit zu verfolgen. Die typischen Amorphi globuli, deren venöser Abfluss in der Mitte des Körpers liegt, werden im Allgemeinen am gleichmässigsten und dabei doch am wenigsten, die nicht typischen Amorphi globuli je nach dem Ort ihres ersten Stromhindernisses und je nach dem Ort der sich entwickelnden Ersatzvenen entweder in ibren unteren oder oberen oder auch mittleren Theilen vollkommener entwickelt werden. Das Oedem kann dabei ziemlich einseitig auftreten, wie bei Fall Splied, Bd. 58, S. 99, und Tafel IV, und es kann dadurch eine Ungleichmässigkeit der äusseren Gestalt entstehen, welche eine Zwischenstufe darstellt zwischen den Amorphis und den Acephalis. Man hat sie Mylacephali genannt. In manchen Fällen entsteht solche Form auch dadurch, dass das Gesammtödem ungleichmässig rückgängig wird, so dass nur ein Theil stark ödematös bleibt.

Wenn die Acardie bei den in Rede stehenden Fällen (wo der venöse Abfluss, sei es durch die zu enge venöse Anastomose auf der Placenta, sei es durch das die Acardie veranlassende Stromhinderniss ganz ungenügend ist) erst eintritt, nachdem der Fötus schon weiter entwickelt ist, so sind die Zerstörungen kaum geringer, weil auch dann das Oedem starke Auftreibung und Gangrän erzeugt. Doch selbst wenn Kopf und Theile von den Extremitäten absterben und abfallen und selbst wenn die Eingeweide fast alle schwinden, so bleibt doch wenigstens eine gewisse Gliederung der äusseren Gestalt übrig, und es entstehen gewöhnlich Acephalen mit starkem Oedem und vielfachen Einzeldefecten oder wenigstens Mylacephalen.

Die Zeit des Eintrittes der Acardie, die verschiedene Weite der zuführenden arteriellen Anastomose und die verschiedene Enge der abführenden venösen Anastomose geben allerlei Uebergänge und Modificationen, die einzelu durchsprechen überflüssig ist.

Es ist auch garnicht nöthig für alle solche Uebergangsformen ein wortreiches Namenregister zu schaffen.

Es genügt, wenn man etwa, wie schon bisher, unterscheidet:

1. Acardii completi (event. mono-, abrachii und mono-, apodes), welche Kopf und Rumpf besitzen (freilich boide oft nicht vollständig). Will man nach einer gewissen Unvollständigkeit des Kopfes noch weiter eintheilen, so kann man sie (nach SaintHilaire) spalten in: 


$$
\begin{array}{ccc}
\text { Acardii completi holocephali, } \\
(n & n & \text { paracephali }), \\
n & n & \text { omacephali), } \\
n & n & \text { hemicephali. }
\end{array}
$$

2. Acardii acormi, welche nur den Kopf besitzen und von Rumpf und Extremitäten höchstens Spuren. Man kann sie wieder spalten in:

$$
\begin{array}{ccc}
\text { Acardii acormi holocephali, } \\
" n & n \text { hemicephali. }
\end{array}
$$

3. Acardii acephali, welche das Beckenende (untersten Theil des Rumpfes) als Grundstock besitzen, an den sich weitere Theile des Rumpfes - aber nicht auch der Kopf - ansetzen können. Sie spalten sich in di-, mono-, abrachii und di-, mono-, apodes.

4. Acardii amorphi, wolche entweder:

a) als typische (mehr weniger deutlich) die ganze Anlage der Frucht umfassen, dies aber in einem rundlichen Hautsack, aus welchem Kopf und Extremitäten höchstens als Spuren hervorragen oder welche

b) als atypische nur von einem stark rundlich angeschwollenen Theil der Frucht gebildet werden, während die anderen Theile wenigstens stark geschrumpft oder gar abgefallen sind, sodass im Ganzen ebenfalls nur eine rundliche Form resultirt.

Zwischen 3 und 4 kann man noch als Zwischenformen die Peracephali und Mylacephali einschieben, gewissermaassen als stark hydropisch verunstaltete Acephali.

Aetiologisch

1. entspricht der (annähernd) genügenden Ernährung des Acardius durch genügend weite Placentaanastomosen;

2. entspricht der ungenügenden Ernährung des Acardius durch zu geringen Blutzufluss bei (annähernd) genügendem venösen Blutabfluss am Kopfende

gewöhnlich durch die erhaltene gemeinsame Dottervene,

manchmal durch die obere Hohl- und wirkliche Nabelvene, während die untere Hohlvene verschwunden ist,

manchmal durch eine Ersatzuabelvene, welche von der Jugularis kommt und subcutan rerläuft, während die untere Hohlvene verschwunden ist;

3. entspricht der ungenügenden Ernährung des Acardius durch zu geringen Blutzufluss bei (annähernd) genügendem renösen Blutabfluss am Beckenende 
gewöhnlich durch die Burow'sche Ersatznabelvene(n), manchmal durch die wirkliche Nabelvene,

manchmal durch mehrere von unten kommende Ersatznabelvenen;

4a. entspricht der sehr frühzeitigen Verengung der Allantois(Nabelschnur)vene, indem diese erst als zu enges zuführendes Gefäss durch ungenügende Speisung des Herzens von der Placenta her den Tod desselben herbeiführt und nachher als zu enges und von der Mitte abführendes Gefäss sehr starkes Oedem des ganzen Acardius bis zum Untergang der meisten Organe im gemeinschaftlichen Hautsack bewirkt. Diese Amorphi entstehen aus orthomorphen Embryonen. 4b. entspricht der frühzeitigen Entstehung von Acardie durch Verengung oder Verödung der Nabelvene - also bei heteromorphen Embryonen -- unter Beihülfe einer viel zu engen venösen Placentaanastomose.

Die Peracephali und besonders die Mylacephali entsprechen der Entstehungsweise von $4 b$, jedoch bei späterem Eintritt der Acardie.

Eine besondere Stellung in der Morphologie nehmen ein

$$
\text { die Hemiacardii. }
$$

Sie zeigen im Allgemeinen recht vollkommen ausgebildete Körperform. Der lange Bestand und die oft bis zur Geburt fortgesetzte Thätigkeit ejnes, wenn auch nur rudimentären Herzens zeugt davon, dass der Circulationsäquator nur unter stärkerem Kampf bis in die Aorta und oft sogar bis in die Nähe des Herzens vorgeschoben werden konnte. Der arterielle Blutdruck und die Gesammtblutmasse muss während dem im Hemiacardius eine nicht ganz geringe Höhe behalten haben. Es konnten also alle oder nahezu alle Körpertheile unter Circulation erhalten werden. Selbst dann, wenn der Hemiacardius schliesslich durch Herztod noch wirklicher Acardius wird, und wenn man event. das Herz nur noch als abgestorben im Acardius vorfindet, wird dieser doch gewöhnlich noch ziemlich vollkommen ernährt werden, weil der Blutstrom vom gesunden Zwilling her, welcher im Stande war, den Circulationsäquator so weit gegen das unterliegende Herz vorzuschieben, auch im Stande sein wird, noch dessen geringen Circulationsbereich mit zu übernehmen. 
Einige Fälle scheinen allerdings dagegen zu sprechen, dass die Hemiacardii immer relativ sehr vollkommen sind.

Der Acormus von Rudolphi hatte ein Organ, welches dieser Autor für das Herz hielt. Dasselbe könnte wegen seiner Grösse recht wohl auch eine Weile functionirt haben. Der ganz gleich organisirte Acormus von Barkow hatte aber sicher kein Herz. Der äusserst sorgfältigen Untersuchung von Barkow würde ein solches nicht entgangen sein. Wahrscheinlich war also auch im Fall von Rudolphi jenes Organ nicht ein Herz, sondern ein Rudiment des Verdauungstractus. Es sprechen dafür auch manche andere Momente.

Neugebauer (Centralblatt f. Gynäk., 1886, No. 44) will ein einkammeriges Herz sogar bei seinem Amorphus gefunden haben. Es kann das aber auch nur ein Rudiment des Darmtractus gewesen sein. Verbindung mit Gefässen ist nicht nachgewiesen. In einem thierischen Amorphus zeigte solches "Herz" alle vier Abtheilungen eines Wiederkäuermagens (s. S. 82).

Im Gefässsystem zeigen die Hemiacardii zwar die sonderbarsten und complicirtesten Verhältnisse, wie oben in Gruppe $X$ (Bd. 58, S. $115 \mathrm{ff}$.) und XII (Bd. 58, S. $138 \mathrm{ff}$.) gezeigt worden ist. Sie entstehen ja meist aus heteromorphen Früchten. Die Körperformen und einzelnen Organe leiden aber darunter nur theilweise. Für sie ist es im Allgemeinen gleichgültig, ob ihre Ernährung vom eigenen oder vom fremden Herzen her besorgt wird. Nur die Eingeweide, welche schon bei den Mikrocardiis stark einschmelzen (s. Bd. 55, S. 614), thun es hier natürlich wenigstens ebenso oder noch mehr. Im Uebrigen gestalten sich die Verhältnisse im einzelnen Falle gewöhnlich so complicirt, dass es nöthig ist, auf die in Gruppe X und XII gegebenen Analysen einiger Fälle zu verweisen.

Will man die anatomische Ausbildung der Acardii und diejenige ihrer Verwandten gemeinschaftlich möglichst kurz und zugleich möglichst nach ihrer Aetiologie charakterisiren, so kann man sagen:

Eineige Zwillinge mit normaler Anlage bleiben normal, wenn ihre so gut wie immer vorhandene gegenseitige Transfusion in der Placenta (durch den gemeinschaftlichen dritten Placentakreislauf) nicht im Laufe der Schwangerschaft ungleich wird. Geschieht dies, wie nicht selten, durch ungleichmässige Entwickelung der gemeinschaftlichen Transfusionszotten und kann die so entstandene Ungleichheit der gegenseitigen Transfusion nicht durch noch vor- 
handene Placentaanastomosen genügend ausgeglichen werden, so wird zunächst der begünstigte Zwilling Makrocardius mit Polyurie, Polyhydramnie, Nierenhypertrophie ete. Er bleibt aber bei geringeren Graden auch noch extrauterin lebensfähig. Bei höheren Graden wird er krank und würde also selbst dann nicht extrauterin lebensfähig werden, wenn er nicht, wie gewöhnlich, wegen der Polyhydramnie zu früh geboren würde. Der benachtheiligte Zwilling bleibt zwar schlecht ernährt, bleibt aber normal und event. extrauterin lebensfähig.

Ist bei eintretender grosser Ungleichheit der gegenseitigen Transfusion in der Placenta eine Anastomose, welche ausgleichend wirken kann, gar nicht vorhanden, so wird auch der benachtheiligte Zwilling krank und zwar wird er Mikrocardius. Durch die Polyhydramnie des begünstigten werden aber beide Zwillinge schon vor Erreichung des lebensfähigen Alters ausgestossen.

Wird einer der Zwillinge heteromorph - Stromhinderniss in der Nabelvene -, so wird er bei Abwesenheit von ausgleichenden Placentaanastomosen, wenn er zugleich der bei der ungleichen Placentatransfusion begünstigte ist, heteromorpher Makrocardius (Gruppe IV, Bd. 58, S. 28, Taf. II), mit starkem allgemeinen Oedem, Polyhydramnie etc., wenn er aber zugleich der bei der ungleichen Placentatransfusion benachtheiligte ist, heteromorpher Mikrocardius $=$ Psendohemiacardius (Gruppe III, Bd. 58, S. 1, Taf. I). Bei beiden läuft das Blut trotz mannigfacher Missbildungen nirgends in abnormer Richtung. Die Defecte am Fötus sind nur gering.

Acardie mit Umkehr des Blutlaufes in bestimmten Hauptgefässen ist nur möglich, wenn auf der Placenta noch eine arterielle und eine venöse Anastomose vorhanden sind. Entsteht Acardie durch primären Herztod oder durch ein Stromhinderniss in der Placenta oder in der Nabelschnurvene, so ist der betreffende Acardius alṣ aus einem ursprünglich normal geformten Fötus entstanden anzusehen. Entsteht sie aber durch ein Stromhinderniss in der Nabelvene, so ist der betreffende Acardius als aus einem, wenigstens in dieser Gegend abnormen (heteromorphen) Fötus hervorgegangen anzusehen. Die Acardie braucht aber beide Male ausser dem Untergang des Herzens und der nächsten Eingeweide (Lungen, Leber) weitere Defecte nicht zur Folge zu haben.

Die vielfachen und verschiedenen starken Missbildungen und Defecte, welche wir an den meisten Acardiis finden, sind nicht Folgen der Acardie an sich, sondern Folgen der ungenügenden 
oder unzweckmässigen Blutcirculation, welche nach Eintritt der Acardie durch die meist dazu ungenügenden Anastomosen auf der Placenta bewirkt wird.

Bei genügend weiter arterieller und renöser Anastomose bleibt der Acardius bis auf Defect von Herz und Umgebung vollständig.

Eine zu dünne arterielle Anastomose kann nur einen Theil des Acardius lebend erhalten. Welcher Theil dies ist, wird durch den so häufig veränderten Ort des (genügenden) venösen Abflusses bestimmt (Acormi, Acephali).

Ist, wie so häufig, die venöse Anastomose auf der Placenta zu eng, so wird zwar durch Stauung ein grösserer Theil des Acardius unter Circulation erhaiten und ernährt, als bei weiter venöser Placentaanastomose. Das mit der Stauung verbundene Oedem zerstört aber wieder wenigstens eben so viel und formt den Acardius, wenn die Acardie früh eintritt, zu einem Amorphus, wenn später, zu einem Mylacephalus oder stark ödematösem Acephalus. Ebenso ist es, wenn erst Acardie und dann ungenügender venöser Abfluss vom Acardius durch ein und dasselbe Stromhinderniss (meist in der Nabelschnurvene) erzeugt werden.

\section{Die Placentaverhältnisse der Acardii.}

Die Placentaverhältnisse dẹr Acardii sind in den vorhergehenden Abschnitten allerdings schon wiederholt berührt worden. Wegen der Wichtigkeit derselben für weitere Forschungen aber scheint es mir doch nöthig, dieselben bier noch im Zusammenhang zu besprechen. Es wird dabei manches zu erwähnen sein, was bisher noch nicht oder nicht genügend zur Geltung gekommen ist.

Man hat auf die Verhältnisse der Placenta und der Gefässe derselben in der früheren Zeit offenbar zu wenig, in der !etzten Zeit aber wieder zu viel Gewicht gelegt. Es ist sicher. zu bedauern, dass wir bei der grössten Anzahl der uns in der Literatur überlieferten Acardii die Placenta-Verhältnisse nicht kennen, und man muss bestrebt sein, bei allen künftigen Beobachtungen die Placenta zugleich mit dem Acardius zu erhalten, und sie ebenso gut wie diesen zu untersuchen. Man würde aber zu viel hoffen, wenn man erwartete, durch eine sehr viel grössere Sammlung von Acardius-Placenten, als man sie jetzt hat, die Aetiologie der Acardie am besten und vollständigsten erforschen zu können. Die Placenta wird uns bei jedem Falle mithelfen, ihn seiner Aetiologie 
nach schneller und genauer zu rubriciren. Sie wird uns aber zumeist die Aetiologie selbst nicht angeben, denn

1. liegt die Ursache der Acardie bei der grösseren Anzahl der Acardii überhaupt garnicht in der Placenta. Wahrseheinlich bildet diese Anzahl sogar die sehr viel grössere Hälfte aller Fälle.

In allen solchen Fällen kann die Placenta natürlich nichts aussagen. Man wird an einem und demselben Fall gewöhnlich nicht 2 Ursachen für die Acardie finden und finden wollen. Man könnte sich also sogar ersparen, die Placenta überhaupt auf den Grund der Acardie hin zu untersuchen in allen denjenigen Fällen, wo solcher in dem Acardius selbst oder in seiner Nabelschnur gefunden wird. Man wird sie aber des allgemeinen Ueberblicks wegen trotzdem stets auch in solchen Fällen mit untersuchen.

2. Auch in denjenigen wenigen Fällen, in welchen die Ursache der Acardie wirklich in der Placenta liegt, wird dies kaum je noch in der Zeit an derselben zu erweisen sein, in welcher sie uns zu Gesicht kommt. Die Placenta und die Gefässe derselben haben sich vom Eintritt der Acardie ab bis zur Geburt gewöhnlich so sehr den neuen Verhältnissen angepasst, dass der frühere Zustand nur in einzelnen, sehr seltenen Fällon sicher zu erkennen ist.

Wollte man sich also bezüglich der Aetiologie der Acardie nur oder vorzugsweise auf die Placenta stïtzen, so würde man jene nie genügend vollständig ergründen können.

Trotzdem will ich nicht unterlassen, die mir zugängigen Acardiusplacenten einer zusammenfassenden Kritik zu unterwerfen.

Zunächst giebt es Acardiusplacenten, denen man auf den ersten Blick ansieht. dass sie aus ganz gewöhnlichen eineiigen Zwillingsplacenten entstanden, und demnach an der Acardie ganz unschuldig sind.

Acardiusplacenten, welche von gow öhnlichen eineijgen Zwillingsplacenten nicht abweichen.

Dazu gehört die Placenta des

Falles von Eysell (Bd. 55, Taf. XXIl, Plac. 37).

Sie ist ganz symmetrisch construirt und erst durch und nach primären Herztod des Acardius auf dessen Seite verödet und stark oingeschrumpft. Sie ist oben Bd. 55, S. 539, genauer analysirt.

Nahezu ebenso deutlich liegt das Verhältniss bei dem

Fall von Astley Cooper (Guy's Hospital Reports I, 1836. p. 227). 
Diese Placenta ist sehr gut injicirt und abgebildet. Die Nabelschnurarterie des Acardius wird von einem Zweig dritter Ordnung. abgegeben, und man braucht sich dieselbe mit den nächsten Arterien nur grösser vorzustellen, so erkennt man in ihnen die Hauptverzweigung der Placentaarterien des Acardius vor Entstehung der Acardie in der bei eineiigen Zwillingen ganz gewöhnlichen Form. Ebenso ist es bei der Nabelschnurvene, deren Verbindung nach dem normalen Mitzwilling hin besonders gross ist. Die Schnur des Acardius ist zwar excentrisch, diejenigen des normalen Zwillings fast centrisch inserirt. Würde aber nicht Acardie eingetreten und würde die Placentahälfte des Acardius normal weiter entwickelt worden sein, so wären die Nabelschnurinsertionen jedenfalls sogar noch ganz symmetrisch geworden, was für eine gewöhnliche eineiige Zwillingsplacenta nicht einmal nöthig ist.

Wie sehr die Placentahälfte, welche zu einem abgestorbenen Herz gehört, einschrumpft, selbst wenn ihre Blutcirculation vom fremden Herzen her weiter unterhalten wird, zeigen die Placenten $D_{8}$, Bd. XXIV, Taf. V, und Plac. 38, Bd. 55, Taf. XXIII.

Der Aeardius bestand aus Rumpf und einem Bein mit einem Rudiment eines zweiten Schenkels. Oben (Bd. 58, S. 68) ist schon gezeigt worden, dass die Ursache der Acardie im Fötus lag. Es waren beide Burow'schen Venen und eine Hautvene zu Ersatznabelvenen erweitert. Das Strombinderniss hatte sich also in der Lebergegend der Nabelvene entwickelt. Es wäre thöricht, in der Placenta noch eine zweite Ursache der Acardie zu suchen.

Bei der Placenta des

Falles Spliedt (Diss. Kiliae 1859)

muss man schon nach der etwas schematischen Zeichnung der injicirten Placenta annehmen, dass ein kleiner Theil der Placenta dem Acardius angehört hat und verödet ist. Die Nabelschnurinsertionen sind nahezu symmetrisch excentrisch. Der Acardius giebt aber noch weiteren Aufschluss. Er ist ein atypischer Amorphus mit grösserer Ausbildung der oberen Rumpfhälfte und eines Koptrudiments (Unterkiefer), s. Bd. 60, Taf. IV. Aus der Beschreibung seines Gefässsystems lässt sich mit ziemlicher Sicherheit erkennen, dass die Ursache der Acardie in der Nabelvene des Acardius zu suchen ist. Es war die linke Vena mammaria externa als Ersatznabelvene entwickelt (s. S. 99). Sicherlich hat also die Placenta keine Schuld an der Acardie. 
So giebt es noch eine ganze Reihe von Acardiusplacenten, an denen man entweder selbst, oder an deren Acardiis man ebenso schnell und sicher wie an den genannten drei erkennen kann, dass sie die Acardie nicht verursacht haben.

Bei maneher anderen Placenta freilich kann man dies nur vermuthen.

Bei denjenigen Acardiusplacenten, deren Schuldlosigkeit an der Entstehung der Acardie man ohne Weiteres weder an ihnen selbst noch an ihren Acardiis erkennen kann, wird man künftig untersuchen müssen, ob nicht besondere bei ihnen vorhandene Eigenthümlichkeiten darüber etwas Aufschluss geben, bezw. ob nicht mindestens unter bestimmten Verhältnissen der Placenta Acardie leichter bezw. öfter eintritt.

Die Nabelschnurinsertionen bei den Acardiusplacenten.

Manche Autoren wollen eine besonders grosse Entfernung der beiden Nabelschnurinsertionen oder wenigstens die velamentöse Insertion der einen Nabelschnur verantwortlich machen. In solchen Fällen soll die Allantois des einen Zwillings nicht genügend Theil erhalten haben an der Placentainsertion, und dadurch soll der betreffende Zwilling mangelhaft ernährt worden und dadurch sein Herz unterlegen sein.

Andere wollen gerade die unmittelbare Vereinigung der Nabelschnüre für ein die Entstehung von Acardie besonders begünstigendes Moment ansehen. Ahlfeld will sogar einen Parallelismus finden zwischen der Entfernung der Insertion der Nabelschnur des Acardius von derjenigen des normalen Zwillings und der Selbstständigkeit des Acardius.

Ich habe bereits oben in der Kritik das Fehlerhafte solcher Ansichten theoretisch gezeigt. Man kann aber auch bei statistischer Zusammenstellung nicht finden, dass bei Acardiusplacenten die Insertionen der Nabelschnüre irgend häufiger sei, es weit auseinander oder nahe an einander liegen, sei es öfter velamentös wären, als es an gewöhnlichen eineiigen Zwillingsplacenten der Fall ist.

Die Acardiusplacenten zeigen bezüglich der Nabelschnurinsertionen geradezu alle Verhältnisse, welche überhaupt an eineiigen Zwillingsplacenten gefunden werden können, und auch nicht in anderem Verhältniss als bei diesen.

Es findet sich 
dichotomische Theilung der Nabelschnur (mit einem Amnion) bei Fall Mery (Hist. d. l'Acad. Paris 1722), Fall Schatz I. (Taf. VI, Bd. 58, Fig. 47),

Fall Breus (Wiener med. Jahrb., 1882, S. $57 \mathrm{ff}$. )

Insertion dicht bei einander (und marginal) bei

Fall Busch (Progr. Marburg. 1875) ein Amnion,

Fall Schatz II (Taf. VII, Bd. 58, Plac. 47),

Insertion $3 \mathrm{~cm}$ von einander bei

Fall Sippel (Diss. Marburg. 1875),

Insertion symmetrisch excentrisch bei

Fall Astley Cooper (s. oben),

Fall Eysell (Diss. Marburg u. Taf. XXII, Bd. 55, Plac. 37)

Insertion symmetrisch marginal bei

Fall Schönborn (Diss. Berl.),

Insertion marginal und gegenüber velamentös

Fall Schatz III (Taf. VII, Bd. 58, Plac. 48),

Insertion symmetrisch velamentös bei

Fall Caseaux (Mém. de la Soc. de Biol. III. 211) ein Amnion,

Insertion central und excentrisch bei

Fall Spliedt (Diss. Kiliae),

Insertion central und marginal bei

Fall Barkow

Insertion central und velamentös bei

Fall v. Rogues (Diss. Narburg)

Fall Mayer (Verh. d. geburtshilf. Ges. Berlin I. S. 132) ein Amnion,

Insertion excentriseh und marginal

?

Insertion excentrisch und velamentös bei

Fall Meimaroglu (Diss. Hal.),

Fall Winslow (Phil. med. Times. 1872. July) ein Amnion.

Es sind also nicht einmal asymmetrische Insertionen der Nabelschnüre besonders oft vertreten. Wäre dies aber auch der Fall, so würde dies in manchen Fällen ebensowohl eine Folge der partiellen Verödung des dem Acardius zugehörigen Theiles der Placenta sein können, wie eine Folge der ursprünglichen Anlage.

Allerdings scheint, wenigstens relativ, öfter ein einziges Amnion gefunden zu werden als zwei. Ich erkläre mir das aber nicht dadureh, 
dass bei Zwillingen mit einem Amnion gewöhnlich auch die Nabelschnurinsertionen einander nahe liegen, wobei dann die Anastomosen öfter kurz und weit sind. Dadurch wird freilich in Wirklichkeit mancher Zwilling bei primärem und manchmal wohl auch bei secundärem Herztod zum Acardius werden, der bei langen und engen Anastomosen vollständig absterben würde. Solcher Grund würde aber für alle Fälle mit nahen Nabelschnurinsertionen, und nicht nur für solche mit einfachem Amnion gelten müssen. Ich erkläre mir dagegen die grössere Disposition der Fälle mit einem Amnion zum Eintritt von Acardie aus der Neigung solcher Fälle zu Situs transversus des einen Zwillings (s. oben). Bei Situs transversus, besonders wenn er partiell ist, tritt in dem betreffenden Zwilling leicht unregelmässige Gefässbildung ein, und dann auch Neigung zu Missbildung des Herzens oder wenigstens Störung in der venösen Blutzufuhr. Auch bei der

Verästelung der an den Placentaanastomosen direct betheiligten Gefässe

werden bei den Acardiusplacenten alle möglichen Verhältnisse beobachtet. Bei der Acardiusplacenta von Hempel (Diss. Hafniae 1850) geschieht die Verbindung je von Arterie und Vene gleich bei der gemeinschaftlichen (marginalen) Insertion der Nabelschnüre. Ebenso bei dem Fall von Breus. Nur ist hier die Insertion central (in der Amnionwand). Der Fall von Hempel ist noch bemerkenswerth dadurch, dass der normale Zwilling nur eine, der Acardius aber zwei Nabelschnurarterien hat, während das Verhältniss sonst immer umgekehrt ist. Ich weiss für diese Abweichung bis jetzt eine Erklärung nicht.

Manchmal beschreibt die verbindende Arterie einen grossen Weg, manchmal die verbindende Vene. Beiderlei verbindende Gefässe geben manchmal $Z$ weige in die Placenta ab (Maimaroglu, Eysell), manchmal nicht. Beides wird gefunden bei dem Falle von Sippel (Dissert. Marburg 1875). Da inseriren die Nabelschnüre $3 \mathrm{~cm}$ von einander entfernt auf der Placenta. Die Arterie des Acardius senkt sich nicht in die Placenta ein, sondern verläuft im weiten Bogen oberflächlich ohne sich zu verästeln, um sich an der Insertion des andern Nabelstranges mit einer Arterie desselben zu verbinden. Die Vene des Acardius läuft aber nahezu direct nach ihrer Ankunft auf der Placenta oberflächlich auf dieser zur Nabelschnurvene des normalen Zwillings, ramificirt verschiedentlich und senkt 
sich in das Placentagewebe ein. „Es lässt sich dies sicher constatiren, obwohl die Injection nicht gelang. Es kommt also dem Acardius hier auch ein Antheil an dem Capillarsystem der Placenta zu, aber freilich nur in dem Sinne, dass er sein Blut hinein ergiesst, nicht aber solches daraus erhält."

Diese grosse Verschiedenheit der Verbindung der Nabelschnurgefässe des Acardius mit den Placentagefässen des normalen Zwillings, welche so gut wie alle Möglichkeiten umfasst, ist freilich auch ganz natürlich. Sie entspricht nur derselben ïberaus grossen Verschiedenheit, nach Lage und Art, welche wir an den arteriellen und venösen Placentanastomosen an der gewöhnlichen eineiigen Zwillingsplacenta gesehen haben. Diese Verschiedenheit der Verbindung sagt also über die Aetiologie der Acardie nichts aus - oder höchstens: dass die letztere mit der ersteren wenigstens gewöhnlich nichts zu thun hat. Am längsten sind die verbindenden Gefässe zwischen beiden Nabelschnüren gewöhnlich offenbar in den Fällen, bei welchen der Acardius eine besondere, von der Placenta des normalen Zwillings getrennte Placenta hat. Diese Fälle bilden wegen dieser Eigenschaft eine ganz besondere Gruppe. Leider ist das Gefässsystem bei ihnen nur einmal genügend untersucht. Ahlfeld hat mit Unrecht diese Fälle als nicht genügend erwiesen discreditiren wollen.

Fälle mit besonderer Placenta des Acardius.

Es ist von früher ganz bekannt, und ich habe es bei der Darstellung der Entwicklung der Placenta (s. dieses Archiv, Band XXVII, S. $12 \mathrm{ff}$.) wieder gezeigt, dass sogar ein Einling zwei Placenten, ja noch mehr haben kann (s. Bd. XXVII, Taf. I, Plac. 30, Taf. II, Fig. III). Ebenso können natürlich auch eineiige Zwillinge zwei Placenten und zwar jeder Zwilling seine eigene Placenta haben (s. Bd. XXIV, Taf. IV, Pl. B 13). Ich besitze auch zwei solche Nachgeburten. Die Placentagefässverbindungen sind aber dabei doch ganz dieselben wie bei einer gemeinschaftlichen Placenta.

Wie nun der eine Zwilling einer gewöhnlichen eineiigen Zwillingsplacenta Acardius werden kann, so kann es natürlich auch einer werden, dessen Placenta mit derjenigen des Mitzwillings nicht so innig, sondern nur durch weiter laufende Gefässe verbunden ist. Ich stelle die Fälle ans der Literatur zusammen. 
Es ist in diesen Fällen nicht immer ganz sicher, ob wirklich jedes Mal jeder Zwilling seine eigene Placenta gehabt hat. Vielleicht inserirten auch einmal beide Nabelschnüre $z w i s c h e n$ beiden Placenten, oder sogar auf einer Placenta, während die andere Placenta nur eine grosse succenturiata darstellte.

Fall von Clark (Tiedemann, S. 8).

Amorphus in besonderen Häuten — d. h. Amnion - und mit einem "eigenen Mutterkuchen, welcher neben dem des wohlgebildeten Kindes an der Gebärmutter angesessen hatte." Missgeburt oval, 4 Zoll lang; 3 Zoll breit; Nabelstrang 11/2 Zoll lang. Vor der Zergliederung des Amorphus wurden die Gefässe des Nabelstranges des wohlgestalteten Kindes injicirt. Die Injectionsmasse drang leicht in die Gefässe beider Mutterkuchen und selbst in die Substanz der Missgeburt. Im Nabelstrang des Amorphus eine Arterie und eine Vene. Es waren weder Hirn, noch Rückenmark, noch Herz, noch Lungen, noch Baucheingeweide vorhanden mit Ausnahme eines kleinen Darmstückes. Der Fall scheint ein typischer Amorphus globulus gewesen zu sein.

Nach meiner oben gegebenen Hypothese der Acardie wird die Ursache in der primären Stenose der Nabelschnurvene des späteren Acardius gelegen haben. Die Placenta selbst war dann natürlich an der Acardie unschuldig.

Fall von A. Monroy (Transactions of the Royal Society of Edinburg. Vol. III. 1794. p. 215. - Tiedemann, S. 22. Elben, S. 41).

"Monstrum hoc suas habebat membranas, placentam propriam cum funicolo umbilicali breviori." "Funiculus umbilicalis . . . . venam continens duasque arterias sanguinis rubri. Vena umbilicalis utramque simul arteriam lumine superabat, atque jam in abdominis introitu in multos discessit ramos, ad omnes corporis partes decurrentes. Arteriae umbilicales venam ubicumque concomitabantur."

Da der betreffende Acephalus nach der guten Beschreibung sicher kein Herz hatte, also sicher ein eineiiger Zwilling war, so können die "Membranae" nur als Amnion aufgefasst werden. Ahlfeld (Berichte und Arbeiten, 1881-82, S. 276) nimmt an, dass Placenta propria nur einen Theil der gemeinschaftlichen Placenta bedeuten soll. Es könnte das allerdings so sein. Es liegt aber auch gar kein Grund vor, der dafür spräche, dass der Acardius nicht wirklich eine eigene Placenta hatte. Der Fall beweist 
sogar in den Einzelheiten seiner sonstigen Beschreibung, dass er durchaus wahr ist. Es liegt hier als Ursache der Acardie offenbar primärer Herztod vor. Die starke Verzweigung der Nabelschuurvene gleich beim Eintritt in den Körper ohne deutlich präponderirende Collateralen beweist, dass nicht ein allmähliches $\mathrm{Ab}$ sterben des Herzens durch Verengung der Allantoisvene oder desgl., sondern dass plötzlicher Herztod eingetreten war. Der Fall entspricht der Entstehungsweise nach ganz dem in der Gruppe I (dieses Archiv, Bd. 55, S. $535 \mathrm{ff}$.) ausführlich analysirten Fall ron Eysell. Er unterscheidet sich von ihm nur dadurch, dass die Placenta des Acardius noch deutlicher getrennt, und wahrscheinlich auch unter dauernder Circulation gehalten, also nicht verödet war. Der Acephale zeigte fast ganz die Defecte wie bei dem Fall ron Eysell. Jedoch kam es bei ihm wegen seiner viel bedeutenderen Circulationsgrösse nicht zur Tösung der Diaphysen ron den Epiphysen, nicht zur Verfettung der Schenkelmuskeln etc.

Fall ron Giel.

Eine besondere PJacenta ist auch bei dem Acephalus von Giel gezeichnet (Elben, De acephalis sive monstris corde carentibus. Berolini 1821. p. 24. Taf. V). Die Zeichnung ist freilich nur schematisch und unproportional. Doch kann man sich nicht vorstellen, dass eine solche Zeichnung gegeben worden wäre, wenn eine besondere Placenta nicht dagewesen wäre. Der Fall ist für weitere Schlüsse zu unvollkommen beschrieben.

Fall von Gluge.

Eine besondere Placenta hatte auch "le monstre amorphe" von Gluge (Sur un monstre amorphe, Journal de méd. de Bruxelles. 1859. Mai. p. 517). Sie war der Placenta des normalen Zwillings juxtaponirt. Das Herz des Amorphus war ein gerader Canal, ans welchem nach unten zwei Arterien abgingen und nach oben eine Vene entsprang. Sein Muskel war ausgebildet, bestand aus quergestreiften Muskeln - die einzigen im ganzen Amorphus. Da mir die Originalbeschreibung nicht vorlag, kann ich über die Ursache der Acardie etc. Genaueres nicht angeben.

Fall von Vrolik (Atlas, Taf. 47, Fig. 1).

Noben normalem weiblichen Zwilling ron 8 Monaten ein Acephalus. Von jenem ist direct gesagt: "Ejus placenta fuit separata a placenta acephali. . Duae sunt arteriae umbilicales ... Tena umbilicalis propter defectum organorum, in quae caeterum dispergitur, nempe hepatis et cordis, in duos finditur ramos, quorum 
alter in superiorem, alter in inferiorem corporis partem dispergitur."

V. glaubt, dass der Blutlauf in der Nabelschnur der normale war. Einen Grund giebt er nicht an.

Vielleicht liegt auch hier primärer Herztod vor; möglicherweise aber auch doppelte Ersatznabelvene für die obliterirte Allantoisvene der Lebergegend, also secundärer Herztod, dessen Ursache dann nicht in der Placenta, sondern im Acardius lag.

Fall von Gourraigne (Tiedemann, S. 12).

Nachdem ein normales Kind mit der normalen Nachgeburt geboren war, kam ein Acephalus von 8"7 "“ Länge und 7 Unzen Gewicht. Die Placenta desselben war weiss, der Nabelstrang von gewöhnlicher Länge und Dicke, mit einer Vene und zwei Arterien. Die Vene theilte sich in zwei grössere Aeste, welche zur Beckenwirbelsäule liefen, wo sie sich allseitig verzweigten. Die Arterien verhielten sich analog.

Ahlfeld ${ }^{1}$ ) will diese Angabe über den besonderen Fruchtkuchen des Acardius nicht gelten lassen, weil die Beobachtung von einem Landehirurgen 1739 gemacht ist. Dieser Einwand könnte nur dann berücksichtigt werden, wenn der Fall wirklich der einzige wäre.

Wahrscheinlich liegt auch hier, wie im Fall Eysell, primärer Herztod vor. Es ist wenigstens sehr zweifelhaft, ob man die zwei grösseren Aeste der Vene, welche zur Wirbelsäule liefen, für zwei Rami venosi anastomotici umbilico-iliaci, also erweiterte Burowsche Venen und somit für zwei Ersatzvenen des Lebertheiles der Nabelvene auffassen kann. In diesem Falle würde die Ursache der Acardie in der primären Obliteration der Allantoisvene der Lebergegend zu suchen sein. Da sich aber die beiden Aeste an der Wirbelsäule gleich allseitig verzweigt haben, so sind dieselben wahrscheinlich keine Ersatznabelvenen, sondern eine von ihnen ist die Nabelvene selbst gewesen. Die Acardie ist dann nicht durch Obliteration der Nabelvene, sondern durch primären Herztod entstanden. In beiden Fällen ist die Placenta an der Acardie unschuldig. Dass die Placenta des Acardins als weiss bezeichnet wird - also verödet war - spricht nur für die Richtigkeit der Beobachtung. -

Aus dieser Aufzählung hat man allerdings den Eindruck, dass Acardie bei getheilter Placenta eineiger Zwillinge relativ häufiger

1) Berichte und Studien. 1881-82. S. 276 . 
vorkommt, als bei nicht getheilter. Da aber bei einer Anzahl der aufgeführten Fälle die Ursache der Acardie nachweislich im Fötus lag und nicht in der Placenta, so bleibt doch noch zweifelhaft, ob überhaupt und eventuell wie oft etwa die Theilung der Placenta an der Acardie Schuld trägt. Man kann sich solche Schuld vorstellen in Form der gar zu langen Gefässverbindungen zwischen beiden Placenten oder anch nur so, dass die Nabelschnurgefässe des einen Zwillings unverhältnissmässig länger sind, als die des anderen, sodass bei ihm leicht länger dauernde Circulationsstörungen und dadureh Herzschwäche bis Herztod entstehen können. Die bei den abgesonderten Acardius-Placenten öfter beobachtete Verödung giebt keinen Aufschluss. Selbst wenn sie sich häufiger als bei nicht abgesonderten fände, so würde sie doch nur als secundär aufzufassen sein, erzeugt durch die längeren und schwierigeren Gefässverbindungen mit der Hauptplacenta.

Es ist zweifellos, dass Acardie auch durch die Gefässverhältnisse der Placenta verursacht werden kann, wie ich oben schon in Gruppe X (Hemiacardii), Bd. 60, S. $115 \mathrm{ff}$, und in Gruppe IX (Bd. 60, S. $105 \mathrm{ff}$.) bei Besprechung des Falles de la Faille dargelegt habe. Doch habe ich dort auch schon erwähnt, dass ich noch keine Acardiusplacenta gesehen habe, bei der es gelänge, den sicheren Beweis dafür zu erbringen. Die Gefässverhältnisse der Placenta sind bei der Geburt seit Eintritt der Acardie gewöhnlich so stark verändert, dass eine sichere Reconstruction derselben und sichere Schlïsse daraus nicht mehr möglich sind. Jedenfalls braucht man zu solchem Versuche immer auch eine genaue Untersuchung des Acardius selbst und der Nabelschnur, und zwar dies alles nach einer gut gelungenen Injection.

Sicher aber sind solche Acardii, welche durch die Gefässverhältnisse der Placenta entstehen, äusserst selten. Unter keinen Umständen kann man im Allgemeinen einen Parallelismus finden zwisehen der Entfernung der beiden Nabelschurinsertionen oder auch der beiden Placenten und der Häufigkeit der Acardie.

Dagegen muss diese Entfernung im Allgemeinen eine deutliche Einwirkung auf die anatomische Ausbildung der Acardii ausüben, weil mit ihr die Wahrscheinlichkeit wächst, dass die Anastomosen, welche noch den Acardius ernähren, von Placentagefässen höherer Ordnung 
abgehen bezw. in solche treffen, und damit von ungenügender Stärke sind.

\section{Die Acardie bei den Thieren.}

Je weniger Ausbeute wir im vorigen Abschnitt aus den menschlichen Acardiusplacenten erhatten konnten, um so näher liegt es zur Erklärung der Aetiologie der Acardie, so weit sie sich von der Placenta herleitet, die Beobachtungen von denjenigen Acardiis heranzuziehen, welche sich bei den Thieren finden.

Wenn auch die Einzelbeobachtungen bei den Thieren meist nicht annähernd so ausführlich gemacht und mitgetheilt sind wie beim Menschen, so bietet doch schon das verschieden häufige Vorkommen der Acardii bei den verschiedenen Thiergattungen manche Anhaltspunkte. Besonders sind es die bei den verschiedenen Thiergattungen ganz verschiedenen Verhältnisse der Nabelschnur und der Placentaanastomosen, welche einigen Aufschluss versprechen ${ }^{1}$ ).

Die beim Menschen schon von der ersten Zeit der Entwicklung her stets einfache $\mathrm{Nabelschnurvene}$ ist bei den Thieren regelmässig und dauernd doppelt. Sie ist ausserdem bei allen Thieren stets viel kürzer und gewöhnlich weniger gewunden als beim Menschen. Starke Verengerung oder gar Verschluss des Rückweges des Blutes von der Placenta zum Zwilling im Verlaufe der Nabeischnur wird deshalb bei Thieren viel seltener vorkommen als beim Menschen.

An der Placenta bleiben bei den Einhufern mit ihrer holochorialen Placenta die reichlichen Anastomosen fast alle nicht allein bestehen, sondorn auch von beträchtlichem Caliber (s. Bd. XXVII, Taf. III, Placenta vom Pferd), bei den Fleisch-

1) Bezüglich dieser Anastomosen habe ich zur Vermeidung von Missverständnissen noch einiges hinzuzufügen. Ioh habe eineiige Zwillingsplacenten von Thieren zu untersuchen bisher keine Gelegenheit gehabt, also die Anastomosen zwischen den Placentakreisläufen beider Zwillinge nicht gesehen. Thre Existenz kann aber nicht zweifelhaft sein. Ohnedem würden ja keine Acardii entstehen können. Ihre Beschaffenheit erschliesse ich aus der Beschaffenheit. der Placenta-Anastomosen am einfachen Ei. Die geringe Tendenz der letzteren zur Verödung bei den Finhufern, die schon grössero bei den Floischfressern, die viel grössere bei den Wiederkäuern und die grösste beim Menschen wird sich sicher ganz parallel auch bei den Anastomosen zwischen den beiden Placentakreisläufen von eineiigen Zwillingsplacenten finden. Controlirende Untersuchungen müssen freilich noch vorgenommen werden. 
fressern mit ihrer zonalen Placenta die arteriellen und venösen Anastomosen innerhalb der Zone der Placenta wenigstens zum Theil bestehen. Nur bei den Wiederkäuern mit ihrer polykotyledonen Placenta veröden die Anastomosen recht reichlich, wenn anch lange nicht so vollkommen wic beim Menschen, und auch die bleibenden werden recht dünn (s. Bd. XXVII, Taf. IV, Placenta rom Rind).

In dieseu rersehiedenen Verhältnissen der Nabelschnurvene und der Anastomosen auf der Placenta beim Nensehen einerseits und den verschiedenen Thiergattungen andererseits kann man gewissermaassen Experimente und Proben der Natur auf meine Theoric erblicken. Wir wollen prüfen, welche Resultate das auf die Thiere bezügliche literarische Material ergiebt.

Dieverschiedene Häufigkeit der Acardii bei den versehiedenen Thiergattungen.

Il. Rieck (Vier Beiträge zur Lehre von den thierischen Missbildungen. Oesterr. Monatsschrift für Thierheilkunde, 1887) hat aus der Literatur 18 Fälle von Acephalus bipes bei Thieren zusammengestellt, und darunter nur 1 von Fleischfressern, 17 von Wicderkäuem gefunden, während von Pferd und Schwein keine gefunden wurden. Zähle ich dazu noch die 7 Fälle von Gurlt in seiner pathologischen Inatomie der Haussängethiere, II, 1832, den von Rieck nicht gezählten Fall von Acephalus tripes aus Gurlt's ,Thierische Missbildungen, 1872" und die Fälle von Amorphus globulus (14) und Acormus (5) aus beiden Arbeiten von Gurlt und 2 Fälle von Vrolik (Amorphus Taf. 46, Acephalus Taf. 47), sowie die 5 Fälle von Tiedemann (S. 46), so crgiebt sich: dass unter 15 Amorphi, 32 Acephali und 5 Acormi, d. i. unter 52 Acardii boi Thieren nur 2 (Acephali) von Fleischfressern herrühten, alle übrigen von Wiederkäuern, keiner von Einhufern oder Sehwein.

Aus dieser Zusammenstellung lïsst sich allerdings nicht allzn viel schliessen. Bei ihr spielen zu viel Zufälligkeiten mit, um mit einigem Recht einer Zusammenstellung der menschlichen Acardii gegenübergestellt zu werden, die selbst wieder ron so vielen, aber andersartigen Zufälligkeiten beeinflusst ist. Ja, es dürfen in der Zusammenstellung der thierischen Acardii nicht einmal die einzelnen Thierarten ohne Weiteres einander gegenüber gestellt werden. So kann man \%. B. einwendon, dass die Wiederkäuer, weil 
zur menschlichen Nahrung dienend, sich einer veil grösseren Untersuchungszahl erfreuen, als die anderen Thiere, besonders die Fleischtresser, dass sie also deshalb allein schon so im Vordergrund stehen können. Trotzdem aber kann man aus der Zursammenstellung doch noch einige recht brauchbare Schlüsse ziehen. So sind z. B. die Pferde, was Pflege und Aufmerksamkeit des Menschen bei ihrer Geburt betrifft, nicht schlechter gestellt als die Wiederkäuer. Dass beim Pferd, wie ich durch weitere briefliche Erkundigungen bei den hervorragendsten Thierärzten festgestellt habe, auch niemals ein Acardius angetroffen wurde und bei den Fleischfressern so ungleich weniger als bei den Wiederkäuern und dem Menschen, muss besondere Ursachen haben. Wir wollen sie suchen.

Vorerst kommen nach Frank (Lehrbuch der thierärztlichen Geburtshülfe, S. 436) Zwillinge, die bei den Wiederkäuern überaus häufig sind, bei Pferden äusserst selten vor. Das Verhältniss wird wohl bezüglich der eineiigen Zwillinge dasselbe oder ähnlich sein. Es müssen also schon deshalb Acardii bei Pferden wenigstens viel seltener sein. Da aber bisher kein Grund vorliegt, anzunehmen, dass beim Pferde eineiige Zwillinge garnicht vorkommen und auch kein Grund, warum dann nicht wenigstens manchmal einer der Zwillinge durch primären oder secundären Herztod absterben sollte, so ist es mehr als wahrscheinlich, ja ziemlich sicher, dass das Nichtvorkommen von Acardii beim Pferde nur scheinbar ist und zwar dadurch, dass dieselben äusserlich wie todte gewöhnliche Früchte aussehen, und deshalh nicht untersucht und nicht als Acardii erkannt werden. Dies erklärt sich daraus, dass beim Pferde das beiden Zwillingen gemeinsame primäre Choriongefässnetz mit seinen allseitigen und weiten Anastomosen dauernd bestehen bleibt. Durch die reichlichen und weiten Anastomosen erbält der horztodte Zwilling, sei es, dass der Herztod primär oder secundär zu Stande kam, sofort vom Mitzwilling vollständige und genügende Blutcirculation, wie etwa der Acardius von Breus (s. Bd. 58, S. 61, Taf. IV, Fig. 41). Er kann deshalb nur wenig oler garnicht zerfallen, behält nahezu seine normale Gestalt und Grösse, entwickelt sogar beide weiter und bleibt bei der Geburt, weil für eine todtgeborene Frucht angesehen, als Acardius unentdeckt. Solche Erklärung kann freilich nicht für das Fehlen auch solcher Acardii gelten, welche durch Verengung bezw. Verschluss einer oder beider Nabelschnurvenen entstehen - Amorphi und 
Acormi -. Es wird uns aber nicht auffallen, wenn gerade solche Acardii beim Pferde wirklich nicht vorkommen. Denn man kann oder muss sogar annehmen, dass bei ihm die Nabelschnurvenen ebenso wenig zur Verengung bezw. Verschliessung neigen, wie die Anastomosen auf der Placenta. Das Pferd würde danach allerdings nur sehr selten, wenn aber, dann auch nur körperlich ganz ausgebildete Acardii aufweisen.

Bei den Fleischfressern mit ihrer zonalen Placenta, bei welchen nur diejenigen Anastomosen des primären Choriongetässnetzes bestehen bleiben, welche auf dieser Zone liegen, und auch diese nur zum Theil, kann es schon eher vorkommen, dass ein primär oder secundär herztodter Zwilling vom normalen Zwilling aus durch die Anastomosen nicht mit genügender Circulation rersehen, und dadurch difform wird. Die meisten solcher Acardii werden aber wohl auch hier äusserlich normale Formen haben, und dadurch, wenn sie nicht etwa vom Mutterthier gleich verzehrt werden, und so dem Menschen überhaupt nicht zu Gesicht kommen, nicht auffallen und nicht untersucht werden. $O b$ auch bei den Fleischfressern die Nabelschnurvenen nicht zu Verengung und Verschluss neigen, wie wahrseheinlich bei den Einhufern, so dass Amorphi und Acormi garnicht vorkommen, muss durch künftige Beobachtungen entschieden werden.

Bei den Wiederkäuern sind die Verbältnisse der Anastomosen auf der Placenta denjenigen beim Menschen am Aehn]ichsten. Die Anastomosen obliteriren zwar durchaus nicht alle. Sie werden aber bezw. bleiben gewöhnlich recht eng. Dieser Aehnlichkeit entsprechend finden sich auch die Acardii bei den Wiederkäuern in ähnlicher Mannigfaltigkeit der Form wie beim Menschen.

Allerdings weist die obige Zusammenstellung aus der Literatur auch bei den Wiederkäuern Acardii mit vollständiger äusserer Körperform nicht auf. Man kennt also bei den Thieren Acardii mit vollständiger äusserer Körperform bis jetzt überhaupt nicht. Dies kommt aber offenbar nur davon her, dass solche Acardii stets als einfache todte Früchte angesehen und daher garnicht untersucht werden. Vorkommen müssen sie ohne $Z$ weilel.

Nimmt man also das Vorkommen von Acardii mit vollständiger äusserer Körperform sowohl bei den Einhufern wie bei den Fleischfressern wie bei den Wiederkäuern als feststehend an, so bekommt man in der Vollständigkeit der Formen, welche die Acardii bei den verschiedenen Thierarten zeigen, eine Scala, welche 
ganz der Entwicklung bezw. dem Bestehenbleiben der Placentaanastomosen entspricht.

Die Acardii sind also bei den verschiedenen Thiergattungen im Allgemeinen in ihrer äusseren Form um so vollständiger ausgebildet, je vollständiger bei diesen die Anastomosen zwischen den Placentakreisläufen beider Zwillinge erhalten bleiben, sodass die Wiederkäuer mit ihren mangelhaften Placentaanastomosen dem Menschen darin am Nächsten stehen.

Bezüglich der Häufigkeit der einzelnen Formen und ihrer Ausbildung lässt sich nur für die Wiederkäuer einiges sagen. Am häufigsten sind die Acephali - in der obigen Zusammenstellung. 26 - darunter 2 von Fleischfressern.

Sie zeigen gewöhnlich recht vollkommene Ausbildung der beiden Rami collaterales venosi umbilico-iliaci (Burow'sche Venen). Es macht den Tindruck, als ob sich diese auch bei den menschlichen Acephalis häufigsten Ersatzvenen für die Nabelvene bei den Wiederkäuern besonders leicht und vollkommen entwickeln, vielleicht weil sie von vornherein weiter im Lumen sind, vielleicht auch, weil die Doppelheit der Nabelschnurvene dies begünstigt. Dadurch gewinnen diese Acephali den Eindruck einer besonders grossen Regelmässigkeit. Man sehe die Abbildungen bei Gurlt, Ueber thierische Missbildungen, Berlin 1877, Taf. I, Taf. II, Fig. 5, Taf. III, Fig. 2. Andere Ersatznabelvenen habe ich an den, freilich nor wenigen, bezüglich ihres Venensystems untersuchten thierischen Acardiis überhaupt nicht finden können. Die Entstehung solcher Acephalen hat man sich nach der oben wiederholt gegebenen Erklärung so zu denken, dass in Folge eines Stromhindernisses in dem Lebertheil der Nabelvene sich die Collateralen nach den Venae iliacae hin (Burow'sche Venen) stärker ausbildeten, und schliesslich bei Verschluss der Nabelvene die alleinigen venösen Verbindungen des Fötus mit der Placenta bilden. Inzwischen ist das Herz in Folge unvollkommener Speisung von der Placenta her schwächer geworden und schliesslich abgestorben, und damit hat sich der Blutlauf in den Nabelschnurgefässen und in den grossen Gefässen des Acardius umgekehrt. Dass dieser dabei nur als Acephalus erhalten bleibt, rührt von der Kleinheit des ihm gewährten Blutstromes $a b$, und diese von den noch bestehenden nicht genügend weiten Anastomosen auf der Placenta.

Der A morphus (globulus) ist nur bei den Wiederkäuern, aber da recht häufig beobachtet (15 unter 46 Acardiis). Gurlt giebt an, 
dass in der Nabelschnur sich immer nur eine Arterie und eine Vene befand, also wie beim menschlichen typischen Amorphus globulus.

Die Entstehung der letzteren habe ich in Gruppe VIlla durch primäre Verengung der Nabelschnurvene erklärt. Diese bewirkt anfangs durch mangelhafte Speisung des Herzens von der Placenta her Schwächung bis Tod des Herzens und später nach Umkehr des Blutstromes in den Nabelschnur- und den grossen Körpergefässen starke Stauung im Acardius mit folgendem sehr starkem Oedem und entsprechenden Verbildungen.

Der primären Verengung der Nabelschnurvene beim Menschen entspricht die Obliteration oder das Fehlen einer der bei den Thieren regelmässig vorhandenen 2 Nabelschuurvenen. Der Ausfall einer Nabelschnurvene beim Kalbe; zu welchem vielleicht oft auch noch eine Verengung der zweiten hinzukommt, hat dann ganz dieselben Folgen wie die Verengung der Nabelschnurvene beim Menschen. Das Fehlen der zweiten Nabelschnurarterie ist secundär wie beim Amorphus des Menschen. Die Neigung der Nabelschnurvenen des Kalbes zur Verengung oder gar zur Obliteration muss wie beim Menschen als analog angesehen werden der Neigung aller Anastomosen auf der Placenta zur Verengung resp. Verschluss. Bei den Einhufern und den Fleisehfressern, bei welchen diese Neigung nicht resp. nur wenig besteht, fehlen deshalb die Amorphi. Der Befund stimmt also mit meiner Theorie.

Vielleicht entsteht eine Anzahl Amorphi bei den Wiederkäuern wie beim Menschen auch durch primären Herztod, wenn dieser erfolgt zu einer Zeit, won die venösen Anastomosen auf der Placenta schon sehr verengt, die Glieder des Embryo aber noch nicht stark ausgebildet sind. In solchem Falle ist dann die Obliteration nicht nur der einen Nabelschnurarterie, sondern anch der einen Nabelschnurvene secundär. Jedenfalls kann es keinem Zweifel unterliegen, dass die Entstehung der Amorphi bei den Thieren im Princip dieselbe ist wie beim Menschen.

Die Entstehung der reinen Acormi habe ich beim Menschen (s. oben Gruppe V, Bd. 58, S. 40 u. Taf. III, Fall von Barkow - also nur Kopf mit Rudimenten des Rumpfes) durch rollständige Obliteration der Allantoisrene im Verlaufe zwischen Placenta und Fötus, also in der Nabelschnur - wenn es um diese Zeit überhaupt solche schon gäbe - erklärt, wobei, resp. wodurch, dann die gemeinschaftliche Dottervene vicariirend als Collaterale 
bei Bestand bleibt. Bei den Thieren würde diesem Vorgang die Obliteration beider Nabelschnurvenen entsprechen.

Die von Gurlt (unter obigen 46 Acardiis) bei den Wiederkäuern aufgeführten 5 Acormi sind anf ihre Richtigkeit nach dieser Richtung nicht zu controliren. Weder Text noch Zeichnung lassen erkennen, woher die Nabelschnurrene stammt. Gurlt selbst sagt über diese Acormi S. 4: "das Aenssere gleicht der ersten Art (Amorphus globulus). Nur ist die Bildung der Knochen etwas weiter vorgeschritten." Möglicher Weise handelt es sich - wenigstens theilweise - um atypische Imorphi mit mehr weniger ausgebildetem Kopf. Am ehesten könnte man daran denken, dass der Pseudo-Acormus subglobulus vom Schaf von Gurlt auf Taf. III (Ueber thierische Missgeburten, Berlin 1877) cin wirklicher Acormus wie beim Menschen sei, dass er also eine Dottervene besässe. Vielleicht sind aber die Acormi der Thiere - wenigstens theilweise - auch Pseudo-Acormi und entstanden durch Obliteration resp. Nichtentwickelung der Vena cara inf. bei Fortbestand der Nabelvene, so dass nur die obere Körperhälfte zur Entwickelung Dabei mag wohl die Obliteration einer Nabelschnurvene öfter mitwirken oder wenigstens gleiehzeitig vorhanden sein. --

Aus dieser Betrachtung der Acardii bei den Thieren ergiebt sich also, so weit die Untersuchungen derselben überhaupt godiehen sind, nichts, was der von mir für die mensehlichen Acardii aufgestellten Theorie widerspräche. Im Gegentheil sind die ActioJogie der Acardie und die Formen der Acardii bei den Thieren im Allgemeinen dieselben wie beim Menschen und die Abweichungen lassen sich erklären aus den Abweichungen, welche die Bedingungen bei den Thieren zeigen.

Primärer Herztod führt bei den Thieren wahrscheinlich gar nicht so selten und auch noch spät zu Acardic. Nur werden die so entstandenen Acardii bei den Einhufern und Fleischfressern nicht erkannt, weil sie wegen der stets noch vorhandenen weiten Anastomosen vom normalen Zwilling her gleich so vollkommen unter Circulation gehalten werden, dass dio bisherigen üusseren normalen Formen beibehalten und fortgebildet werden. Nur bei den Wiederkäuern mit ibren viel kargeren und engen Anastomosen ähneln die Verhältnisse mehr denjenigen beim Menschen. Thre durch primären Herztod entstandenen Acardii verkümmern häufig durch ungenügenden Blutstrom rom normalen Zwilling her, werden also Acephali oder, wenn früh entstanden und stark ödematös, Amorphi. 
Secundärer Herztod und damit Acardie durch denselben wird bei Thieren offenbar viel seltener als beim Menschen durch die Verhältnisse in der Placenta und der Nabelschnur erzeugt wegen der Doppelheit der Nabelschnurvene und wegen des Fortbestandes weiter Placentaanastomosen (besonders bei Einhufern und Fleischfressern). Es bleiben also, abgesehen von den Wiederkäuern, so gut wie allein diejenigen Verengungen des venösen Rückflusses als Ursache des secundären Herztodes übrig, welche im Fötus selbst sich bilden (Nichtentwicklung des Ductus venosus Arantii, Verengung oder Obliteration der Nabelvene in der Lebergegend, Obliteration der Vena cava inferior, Verengung der Nabelvenen im Nabelring?) Theilweise ist sicher die viel grössere Seltenheit der Acardie durch secundären Herztod bei den Thieren auch nur scheinbar, weil die so entstandenen Acardii wegen der weit bleibenden Anastomosen bei den Einhufern wohl immer, bei den Fleisch. fressern gewöhnlich ihre äussere normale Form behalten, also nur für todte Früchte gehalten und als Acardii nicht erkannt werden. Nur bei den Wiederkänern bestehen, soweit bis jetzt Erfahrungen vorliegen, nach Frequenz und Ausbildung der Acardii ähnliche Verhältnisse wie beim Mensehen.

\section{Die parasitären Acardii.}

Ich habe nicht die Absicht, die Aetiologie auch der parasitären, d. l. derjenigen Acardii eingehend zu besprechen, welche keinen Nabelstrang haben, sondern mit dem (gesunden) Mitzwilling körperlich verbunden sind.

lch könnte recht wohl auch über sie eine grosse Abhandlung schreiben. Doch unterlasse ich das. Einmal gehört dieses Thema, genau genommen, nicht mehr in den Rahmen dieser Arbeit, und dann jst solche eingehende Abhandlung, wenn man einmal die Aetiologie der freien Acardii kennt, zu einem gewissen Verständniss der Verhältnisse anch bei den parasitären Acardiis nicht mehr nothwendig. Die freien Acardii beleuchten bis zu einem gewissen Grade auch die parasitären.

Tch gebe also nur die hauptsächlichsten Gesichtspunkte, von welchen aus man auch über die parasitären Acardii genügenden Coberblick gewinnt.

ILeckel (Müller's Archiv, 1850, S. 259) hält die Geschwülste am Krenzbein und am Gaumen für Fälle, wo primäre regelmässige 
Pygopage oder Cephalopage unregelmässig zwillings-asymmetrisch geworden sind.

„Es ist leicht zu begreifen, warum die unregelmässigen Formen häufiger vorkommen als die regelmässigen. Denn Doppelmissbildungen mit zwei Nabeln müssen beide das Bestreben aller Fötus haben sich spiralig an ihren Nabelsträngen zu drehen; dies kann nur durch enge Umschlingung der Stränge geschehen, bei welcher in dem irgend schwächeren Strang durch Torsion und Atrophie leicht die Circulation aufhört oder fast von vornherein nicht zu Stande kommt; ist dann ein Fötus ohne einen Nabelstrangkreislauf, so kann er nur vermöge seiner Verwachsung mit dem siegenden Fötus als dessen Parasit durch Collateralkreislauf erhalten bleiben, wird aber bei der Geburt um so kümmerlicher erscheinen, je früher sein Kreislauf unselbstständiger geworden war. Analogien zu derartigen Vorgängen bilden alle Parasitbildungen an monocephalischen Doppelbildungen, deren Kreislauf parasitisch von dem des Trägers abhängig ward."

Diese Anschauung hat ihre volle Berechtigung. Aber sie ist viel zu einseitig. Es kann allerdings bei verwachsenen $Z$ willingen durch gegenseitige Umschlingung der Nabelschnüre Tod des einen Zwillings eintreten, wie das ja.auch bei freien, in einem Amnion befindlichen Zwillingen oft genug geschieht. Es kann dann, während im letzteren Falle der todte Zwilling sich wie jede andere durch Nabelschnurtorsion abgestorbene Frucht verbält, in ersterem Falle bei genügend guten directen Gefässvcrbindungen zwischen den verwachsenen Körpern beider Zwillinge der durch die Nabelschnurtorsion zunächst nur herztodte Zwilling von dem weiterlebenden her mehr weniger vollkommen unter Circulation gehalten, also weiter emährt und sogar in gewissem Grade fortgebildet werden. Es ist das also eine Entstehung der Acardie ähnlich derjenigen, welche ich bei den freien Zwillingen oben in Gruppe I (Bd. 55, S. 535) als Folge von primärem Herztod besprach. Der Unterschied besteht nur darin, dass sich bei den parasitären Acardiis die weiter ern̈ährenden Gefässe nicht in der Nabelschnur, sondern in der Verbindungsstelle zwischen den zusammengewachsenen Zwillingen befind̉en. Da diese Gefässe - wenigstens bei den Cephalopagen und besonders bei den Pygopagen --- gegenüber denjenigen der Nabelschnur gewöhnlich recht unvollkommen und unsymmetrisch ausgebildet sind, so kann es nicht auffallen, dass Ernährung und Weiterbildung solcher parasitären Acardii gewöhnlich recht mangelbaft ausfallen.

Es giebt aber ausser diesem ron Meckel angegebenen noch versehiedene andere Wege, auf welchen parasitäre Acardii entstehen und zwar noch viel mehr Wege als bei den freien Acardiis, 
weil verwachsene - oder besser gesagt: nicht vollständig getremte Zwillinge cinen gemeinschaftlichen dritten Kreislauf, wic er zur Entstehung von Acardie nothwendig ist, nicht nur im gemeinsamen Choriongefässnetz - und später daraus hervorgehend in der gemeinschaftlichen Placenta -- besitzen, sondern noch einen ebensolchen im gemeinschaftlichen Fruchthof.

Wie sich der gemeinschaftliche dritte Placentakreislauf mit und zwischen den beiden gewöhnlichen Placentakreisläufen aus dem gemeinschaftlichen Choriongefässnetz entwickelt und gewissermaassen aus den massenhaften Gefässverödungen übrig bleibt, so entwickelt sich ganz ähnlich resp. bleibt übrig aus dem gemeinschaftlichen Fruchthof zwisehen den beiden besonderen Körpergefässsystemen auch ein gemeinschaftlicher dritter Körperkreislauf mit allen den gleichen Eigenschaften, welche wir an dem-gemeinschaftlichen dritten Placentakreislauf kennen. Alle Arten von Gefässen, welche ich oben (Bd. 24, S. $354 \mathrm{ff}$.) geschildert habe und welche wir in der ausgetragenen Plerdeplacenta auf Tafel III von Bd. 27 dauernd und gewissermaassen grob gezeichnet finden, sind mutatis mutandis auch im gemeinschaftlichen Fruchthof vorbanden und erfahren ganz analoge Veränderungen. Wie im gemeinschaftlichen Choriongefässnetz, so sind auch im gemeinschaftlichen Fruchthof je zwischen den Arterien und je zwischen den Venen der beiden, jedem Zwilling besonders zugehörigen Blutgefässsysteme zahlreiche (oberflächliche) Anastomosen vorhanden und der zwischen den beiden Blutgefässsystemen sich bildende Circulationsäquator wird ausserdem noch vielfach durchbrochen von capillären Transfusionsströmen, welche Blut aus Arterien des einen Zwillings in Venen des anderen, und von capillären Transfusionsströmen, welche umgekehrt Blut aus Arterien des zweiten Zwillings in Venen des ersten transfundiren. Nach vielfachen Verödungen bleiben wie in der Placenta vom gemeinschaftlichen Choriongefässnetz, so in der Vereinigungsstelle beider Zwillinge vom gemeinschaftlichen Fruchthof je einige capilläre Transfusionsbezirke ïbrig, welche Blut vom ersten zum zweiten und andere, welche Blut vom zweiten zum ersten transfundiren und daneben grössere arterielle Anastomosen, welche Arterien des einen mit Arterien des zweiten, und grössere venöse Anastomosen, welche Venen des einen mit Venen des zweiten verbinden. Es ist sogar nicht unwahrscheinlich, dass die Analogie zwischen dem gemeinschaftlichen dritten Placentakreislauf und dem 
gemeinsehaftlichen dritten Körperkreislauf (in der Vereinigungsstelle beider Zwillinge) noch weiter geht, dass also nicht immer alle 4 Verbindungsarten der beiden Gefässsysteme fortbestehen bleiben so dass die Verbältnisse aller vier Typen der eineiigen Zwillingsplacenten A-D auch in dem gemeinschaftlichen dritten Körperkreislauf ungetrennter Zwillinge vorkommen. (A: nur capilläre gegenseitige Transfusionsströme, B: die gleichen mit einer oder mehreren arteriellen Anastomosen, $\mathrm{C}$ : die gleichen mit einer oder mehreren venösen Anastomosen und D: die gleichen mit sowohl arteriellen als venösen Anastomosen.) Alle Vorgänge nun, betreffend Entstehung der Acardie und verwandter Zustände, welche sich im gemeinschaftlichen dritten Placentakreislauf und in Folge davon in den Zwillingen abspielen, können sich mutatis mutandis ebenso auch im gemeinschaftlichen dritten Körperkreislauf abspielen, also: Acardie des einen Zwillings durch primären Herztod etc. bei der Gefässverbindung des Typus D, Makrocardie bei Typus A und B, Mikrocardie bei Typus A oder C, Hemiacardie bei Typus D (und B).

Dadurch, dass die ungetrennten Zwillinge nicht nur einen gemeinschaftlichen dritten Kreislauf haben wie die freien, sondern zwei, haben sie auch die doppelte Möglichkeit, dass bei einem der Zwillinge Acardie entsteht. Will man noch berücksichtigen, dass die Weiterernährung des Acardius nicht durch denselben gemeinschaftlichen dritten Kreislauf zu erfolgen braucht, mittelst dessen die Acardie eingetreten ist, so wird die Zahl der Möglichkeiten noch erheblich grösser. Wir finden denn auch die parasitären Aeardii relativ und absolut ungleich häufiger als die freien. Vierfach wird die grössere Häufigkeit allerdings wohl nicht, wie man meinen möchte, weil die Asymmetrie, welche etwa bei beiden gemeinschaftlichen dritten Kreisläufen vorhanden ist, sich oft genug gegenseitig mindern oder ausgleichen kann.

An Stelle dieser Abminderung der Häufigkeit kommen freilich umgekehrt wieder zwei Momente hinzu, welche vermehrend wirken.

1. Bei ungetrennten Zwillingen kann Acardie schon durch primären Herzmangel eintreten, d. i. dadurch, dass sich in dem einen Keim ein Herz überhaupt nicht entwickelt, so dass er von vornherein durch den Fruchthof des anderen Keimes und später durch die Vereinigungsstelle beider Zwillinge mit ernährt wird.

2. Die freien eineiigen $Z$ willinge haben nicht immer, sondern wahrscheinlich sogar relativ selten einen gemeinschaftlichen Dotter und 
dementsprechend einen gemeinschaftlichen Dotterkreislauf. Bei den ungetrennten Zwillingen ist er offenbar ungleich häufiger, ja zumeist vorhanden (nur nicht bei den doppelnabligen Doppelbildungen). Ein gemeinschaftlicher Dotterkreislauf birgt in sich stets auch einen gemeinschaftlichen dritten Kreislauf und kann dadurch auch für sich allein Acardie erzeugen. Freilich kann ich dafür einen beweisenden Fall nicht bringen, und ich habe deshalb bei den früheren Auseinandersetzungen diese Ursache der Acardie unberücksichtigt gelassen. Aber in Gruppe $V$ und Gruppe XII habe ich wenigstens gezeigt, wie die gemeinschaftliche Dottervene die obliterirte Nabelschnurvene ersetzen und die verengte ergänzen kann, so dass durch sie mindestens indirect Acardie bezw. Hemiacardie entstehen kann dort, wo ohne sie nur einfacher Tod bezw. Acardie entstehen würde. Jedenfalls also vermehrt der bei den ungetrennten Zwillingen gewöhnlich rorhandene gemeinsame Dotterkreislauf die Häufigkeit der Acardie bei denselben gegenüber der Häufigkeit bei den freien Zwillingen.

Tritt Acardie auf Grund des gemeinschaftlichen dritten Fruchthofes oder des gemeinschaftlichen dritten Dotterkreislaufes v or Entwicklung des Allantoiskreislaufes ein, so kommt dieser letztere bei dem Acardius überhaupt nicht zu Stande. Der Acardius wird je nach der Gefässverbindung zwischen den beiden Körperanlagen mehr weniger vollständig, aber stets als Parasit ausgebildet und weiter ernährt.

Tritt Acardie auf Grund des gemeinschaftlichen dritten Fruchthof- (resp. Dotter)kreislaufes nicht ein und entwickelt sich also auch der gemeinschaftliche dritte Allantoiskreislauf, so werden die beiden danach zu gleicher Zeit bestehenden dritten Kreisläufe in der Placenta und in der Verwachsungsstelle (der Dotterkreislauf schwindet alsbald) beide dynamisch symmetrisch sein können und dadurch wirkungslos bleiben oder beide umgekehrt, aber gleich stark asymmetrisch mit gleichem negativen Erfolge. Ein Erfolg tritt in letzterem Falle erst mit der Geburt ein, wo der asymmetrische dritte Placentakreislauf aufhört und nur der asymmetrische dritte Körperkreislauf übrig bleibt. Seine Asymmetrie wird nach der Geburt für beide Zwillinge recht unangenehm, event. sogar verderblich werden können. Vor der Geburt addirt sich gleichsinnige Asymmetrie beider dritter Kreisläufe, ungleichsinnige subtrahirt sich cte. etc. Da die Asymmetrieen wachsen und abnehmen können, so kommen allerlei weitere Veränderungen vor.

Der Placentakreislauf des einen Zwillings kann durch Schnur- 
torsion oder dergl. schon intrauterin aufhören, wie Meckel hervorhob. Daraus braucht aber nicht unbebedingt Acardie hervorzugehen, sondern kann auch Mikrocardie des benachtheiligten Zwillings entstehen, wemn nämlich der asymmetrische dritte Körperkreislauf gar keine arteriellen und venösen Anastomosen besitzt (analog der Gruppe II). Es ist aber solcher Fall wohl noch nicht gesehen worden. Ebenso ist auch noch kein Fall von parasitärer Acardie gesehen worden, wo der Acardius sowohl durch die Körperverwachsungsstelle, als auch durch die Nabelschnurgefässe weiter ernährt worden wäre. Unmöglich ist aber solcher Fall nicht.

\section{Die anatomische Ausbildung der parasitären Acardii.}

Sie entspricht im Allgemeinen derjenigen der freien Acardii, wenn auch natürlich nicht alle Formen sich gleichen und besonders nicht in gleicher Häufigkeit vorkommen.

Stauungserscheinungen - Oedem, Cystenbildungen - sind bei den parasitären Acardiis gewöhnlich nicht geringer, als bei den freien. Ja, es macht sogar don Eindruck, als ob, wenn auch alle anderen Formen vertreten sind, die Amorphi unter den parasitären Acardiis sogar das Hauptcontingent stellen, was bei den freien Acardiis durchaus nicht der Fall ist.

Wie bei den freien Acardiis, so können auch bei den parasitären nur sehr weite und bequeme Anastomosen den eigentlich dem Absterben gewidmeten zweiten Zwilling ganz unter Circulation erhalten. Bei mangelhaiten Anastomosen bleibt immer ein mehr weniger grosser Theil desselben ausser Circulation und stirbt ab. Bei den Pygopagen und Epignathen sind die Anastomosen gewöhnlich viel unvollkommener als bei den Sternopagen. Daher sind jene so häufig besonders stark verkümmert und entartet.

"Die angeborenen Geschwülste der Kreuzbeingegend", welchen Braune eine Monographie gewidmet hat (Die Doppelbildungen und angeborenen Geschwülste der Kreuzbeingegend, Leipzig 1862) bieten eine ganze Reihe von parasitären Acardiis und könnten sehr wohl gleich als Belege und Beispiele für meine Darlegung benutzt werden. Natürlich muss man sich hüten, auch Missbildungen durch einfache Spaltung der Achse, oder gar wirkliche Geschwulstbildungen mit hereinzuziehen, bei denen allen von Anfang an nur ein Herz da ist.

Ich wähle von den Fällen der Braune'schen Monographie nur einige aus. 
Einen parasitären Acardius acormus stellt jedenfalls der Fall von Chabelard dar (Braune, S. 14). Auf dem letzten Rückenwirbel eines sonst wohlgestalteten Kindes sass ein Kopf mit einem zwei querfingerbreiten Halse, das Gesicht zur Erde gewandt. Näheres ist; weil die Originalmittheilung (Mém. de l'Acad. royale des sciences, 1746, T. 27, p. 68) nicht aufzutreiben gewesen, mir nicht bekannt geworden.

Aber der Fall von Nockher ersetzt den Mangel. Bei diesem Fall Med. Zeitung von dem Verein für Heilkunde in Preussen, VI, 1837, No. 3) hing der Acormus -- ein hydrocephalischer Kopf von der Grösse und Form einer mit Luft gefüllten Ochsenblase und ron einem Gewicht von $14 \mathrm{Pdd}$. - mit einem schmalen und kaum einen halben Zoll langen Halse oder Stiele mit einigen sehnigen Fascru und schwachen Rudimenten von. Halswirbeln am Schwertfortsatz des Brustbeins und den kurzen Rippen der linken Seite des normalen ausgetragenen und ziemlich gut genährten Mitzwillings an. Von den beide verbindenden Gefassen war keines so gross, dass man es hätte für eine Carotis halten können.

Ein parasitärer Acardius acephalus ist jedenfalls der Fall von Farber (Oesterr. medicin. Wochenschrift, 1842, 4. Qu., p. 1120, Braune, S. 17). Das Mädchen trug in der linken Lendengegend bis gegen den Steiss hin ein zweites Becken mit unteren Extremitäten, wodurch die untere Körperhälfte doppelt erschien. Das Becken war dem normalen an Grösse gleich und mit vollen gerundeten Hinterbacken und Hüften versehen. In der Kerbe der Hinterbacken fand sich eine $1 / 2$ " tiefe, blind endigende Afteröffnung. Das Steissbein war deutlich zu fühlen, der Schamberg gerundet, an Stelle der Genitalien nur Hautfalten. Oberschenkel, Kniescheibe, Unterschenkel normal. Klumpfüsse. Das accessorische Becken war durch Bandmasse verschiebbar angeheftet. Das Wachsthum ging im Autositen und Parasiten gleichmässig vorwärts.

Fin mehr allseitig entwickelter parasitärer Acardius (oder gar Mikrocardius?) ist wahrseheinlich der Fall von Berigny (Gaz. médie. de Paris, 1844, XII, p. 518. Arch. génér., 1846, 4. Sér., T. VI, p. 119. - Braune, S. 17). Zweiundzwanzig Tage altes, sonst gut gcbildetes Iädehen, trug am unteren Stammesende cin zweites verkümmertes Individuum. Der Parasit hatte Darm, Afteröfnung, Klumpfüsse.

Es wäre nicht schwer, aus den Zusammenstellungen von Braune noch eine grössere $\Lambda$ nzahl von parasitären Acardiis 
herauszusuchen. Es würde dies aber wenig Nutzen haben, weil die Beobachtungen meist zu unvollständig sind, um die Entstehungsweise sicher nachweisen zu können. In den Atlanten von Förster und Ahlfeld finden wir eine grössere Zahl weiterer Fälle und zwar auch ron kephalopagen und sternopagen Acardiis, und in der übrigen Literatur und als Sehenswürdigkeiten treten immer wieder neue Fälle auf. So ist der im Berliner Panoptikum gezeigte, in der lllustrirten Zeitung vom 27. Juni 1891 abgebildete und ron Virchow am 15. April 1891 in der Berliner med. Ges. (Berl. Klin. Wochenschr., 1891, Nor. 19.) vorgestellte 20jährige Indier Lalso ein Sternopagus acardius acephalus, welchem in seinen äusseren Formen nur der Kopf fehlt.

Die Epignathi sind alle parasitäre Acardii acephali mit mehr weniger grosser Ausbildung, von soleher der Figur 19 und 20 anf Tafel II, Bd. XXX, ab bis zu dem Fall ron de la Faille auf Tafel V, Bd. 60, und noch weiter.

Nur eine Beobachtung möchte ich schliesslich nicht versäumen noch hervorzuheben, welche für die Aetiologie der Acardii von besonderem Interesse ist. Die sternopagen Zwillinge liefern trotz ihrer relativ so grossen Häufigkeit verhältnissmässig wenig Acardii. Bei ihnen sind die Herzen beider Zwillinge stets einander sebr nahe gerückt, und sehr häufig durch grosse Gefässe, nicht selten sogar direct verbunden. Wenn nun, wie nicht selten, das Herz des einen Zwillings wesentlich kleiner und unvollkommener entwickelt ist als das des andern, so wäre damit die beste Gelegenheit gegeben, dass nach der Vorstellung ron Claudius das kräftigere Herz das schwächere durch seine kräftigere Pulswelle zum Stillstand zwänge und Acardie erzeugte. Und doch geschieht dies gerade nicht häufig - ein Beweis, welcher neben allen andern in der Kritik der Hypothesen von Dareste-Panum und Claudius-Ablfeld, vorgeführten die Unhaltbarkeit der Vorstellung von Claudius besonders deutlich nachweist. Nicht die Besehaffenheit des arteriellen Gefässsystems, auf welche Claudius ganz einseitig seine Aufmerksamkeit lenkte, ist das Maassgebende bei der Entstehung der Acardie, sondern die Beschaffenheit des Venensystems. Wird ein Herz genügend vom Venensystem her gespeist, so hält es auch einem vicl grösseren Herzen gegenüber Stand, gerade wie bei einer Wasserleitung recht wohl zwei ungleich grosse Pumpmaschinen zugleich Wasser in ein gemeinschaftliches Reservir pumpen können, ohne dass die kleinere Pumpe still steht, wenn sie nur genug gespeist 
wird und genügend Spannkraft hat. Letztere gewinnt das kleinere Herz selbst bei geringerer Wanddicke entweder durch die stärkere Wölbung seiner Wand - bei geringer Füllung -, oder durch grössere Dehnung seiner Muskelfasern -- bei vermehrter Füllung. - Die ungenügende Speisung des Herzens durch das Venensystem, und damit die Beschaffenheit des Venensystems ist also bei Entstehung der Acardie das wichtigere Moment, ja sogar die Hauptsache.

\section{Die Zeit, in welcher Acardie eintritt.}

Man könnte die Frage, wann Acardie eintreten kann, allerdings sehr kurz dahin beantworten, dass dies in jeder Zeit der Schwangerschaft möglich ist. Denn einerseits kann schon bei der Spaltung eines Keimes in zwei Keime, wenn der Fruchthof gemeinschaftlich bleibt und die Bildung des einen Herzens ausbleibt, der diesem zugehörige Theil des Fruchthofes incl. seines Keimes von dem allein angelegten Herzen aus unter Circulation erhalten und so der Keim als parasitärer Acardius weitergebildet werden. Andererseits kann ein Hemiacardius erst gegen das normale Ende der Schwangerschaft Holoacardius werden, wenn auch sein todtes Herz anatomisch noch. vorhanden bleibt. Und auch in der ganzen zwischenliegenden Zeit der Schwangerschaft kann es keinen Moment geben, in welchem nicht das eine Herz (z. B. einer Doppelmissbildung), sei es durch primären, sei es durch secundären Tod (z. B. auf dem Wege des dritten gemeinschaftlichen Kreislaufes in der Verbindungsstelle beider Körper), zu schlagen aufhören kann, sodass das überlebende Herz die Bluteirculation und die mehr weniger vollständige Erhaltung des herztodten Zwillings mit übernehmen muss.

Solche allgemeine Beantwortung ist aber jetzt höchstens noch für die parasitären Acardii brauchbar, über deren Entstehung wir zunächst nur allgemeine und von den freien Acardiis entnommene Vorstellungen haben. Ueber die Zeit der Entstehung der freien Acardii können wir auf Grund der bisherigen Untersuchungen schon eingehendere Angaben machen.

Im Allgemeinen können auch freie Acardii in jeder Zeit der Schwangerschaft entstehen. Doch sind die einzelnen Formen bezüglich ihrer Entstehung zumeist auf ungleich kürzere Zeiträume beschränkt. So kann allerdings auch ein freier Hemiacardius noch in der zweiten Hälfte der Schwangerschaft, ja bis kurz vor 
der Geburt zum Holoacardius werden, indem der Blutstrom des gesunden Herzens erst so spät bis zum kranken vordringt und es zum Stillstand bringt oder eigentlich richtiger gesagt, indem das Herz des Hemiacardius von seinen Venen her allmählich immer weniger und schliesslich so wenig gespeist wird, dass es einen nennenswerthen arteriellen Druck nicht mehr erzeugen und dem arteriellen Druck des gesunden Herzens Widerstand leisten kann. In so später Zeit der Schwangerschaft wird aber solcher Acardius eben nur dann entstehen können, wenn er durch Hemiacardie schon längere Zeit vorbereitet ist, so dass bei Eintritt der Holoacardie nur ein relativ kleiner Theil des ganzen Gefässsystems noch zu übernehmen ist. Wenn ohne solche Vorbereitung z. B. primärer Herztod einen nahezu ausgewachsenen Zwilling trifft, so wird bei ihm wohl immer alsbald Gesammttod folgen, selbst wenn die Anastomosen auf der'Placenta sehr weit sind.

Es ist ja schon schwer glaublich gewesen, dass bei dem Fall von Eyssel (Gruppe I, Bd. 55, S. 535) die Acardie erst in der Mitte der Schwangerschaft eingetreten ist. Es müssen erst neue und gut bewiesene Fälle gefunden werden, wenn man annehmen soll, dass der gleiche Vorgang wie beim Fall Eyssel auch in der zweiten Hälfte der Schwangerschaft möglich ist.

Die einzelnen Formen der Acardii und ihrer Verwandten resp. ihre Entstehungsweisen vertheilen sich etwa auf folgende Zeiträume:

Hemiacardii können in jeder Zeit der Schwangerschaft entstehen und bis zur rechtzeitigen Geburt als solche fortbestehen, ebenso wie orthomorphe und die heteromorphe Mikro- und Makrocardii. Doch werden alle diese Formen häufig vor dem normalen Ende der Schwangerschaft geboren, weil die Polyhydramnie des begünstigten Zwillings früheren Eintritt der Geburt bewirkt.

Inwieweit Polyhydramnie des gesunden Zwillings auch bei Acardie des anderen eintreten und verfrühte Geburt bewirken kann, muss noch weiter untersucht werden. Wolff hat in diesem Archiv, Bd. 59, einen schönen Fall von Drillingsgeburt mit einem Acardius veröffentlicht und mịt dem gleich schönen Fall von $H$. Meckel verglichen. In beiden Füllen befand sich der eine Drilling in einem besonderen $\mathrm{Ei}$, war also ganz unabhängig von den beiden anderen, und war normal. Es betrug das Herzgewicht des mit dem Acardius verbundenen Zwillings deutlich mehr als dasjenige des Drillings, welcher sich im besondereñ Ei befand, bei dem Fall von $W_{0}$ lff $12,25 \mathrm{~g}: 8,85 \mathrm{~g}$ oder $9,28: \mathbf{7 , 4 4} \%$ des Gesammtkörpergewichtes. Dieser Autor nimmt das Herzgewicht des unabhängigen Drillings als Vergleichsgrösse und findet, dass bei dem mit dem Acardius verbundenen Drilling eine deutliche Herzhypertrophie vorhanden gewesen sei. Allerdings wäre dieser Schluss noch nicht 
zwingend. Denn wir finden in den verschiedenen Eiern derselben Schwangerschaft häufig sehr grosse Verschiedenheiten in der Ernährung. Aber es ist auch ohne solchen Vergleich eine gewisse Herzhypertrophie erweislich. Denn wenn auch das Herzgewicht des Zwillingsbruders des Acardius an Höhe nicht dasjenige der Makrocardii $(10-14 \%$ on $)$ erreicht, so kommt es doch bis an die obere Grenze des Normalgewichts heran, bezeugt also wenigstens wohl eine relative Hypertrophie. Ob dies genügt, auch nur zu häufigst Polyurie und Polyhydramnie zu erzeugen, sodass gewöhnlich Früh- oder Fehlgeburt eintreten müsste, müssen erst weitere Beobachtungen lehren.

Die Acardii acormi entstehen schon zu der Zeit, wo neben dem Allantoiskreislauf noch der Dotterkreislauf besteht, wie aus dem Fortbestand der gemeinsamen Dottervene zu erweiseu ist.

Wenn zu dieser Zeit die Allantoisvene bei den Acormis vollständig obliterirt, so wird sie bei anderen Fällen in der gleichen Zeit mindestens zu eng werden und dadurch - also eben so früh die typischen Amorphi globuli erzengen. Amorphi können aber auf demselben Wege offenbar auch noch etwas später entstehen.

Geschieht jedoch die Verengung der Nabelschnurvene viel später, oder bleiben die frühgebildeten Ersatznabelvenen zu eng, so wird der Acardius nicht mehr kugelig, sondern mindestens ein Paramorphus oder Myacephalus oder gar stark hydropischer Acephalus (Fall von Gerges, Bd. 58, S. 102).

Acardie durch Verengung bis Verschluss der Nabelvene zumeist in der Lebergegend oder auch nur durch Nichtentwickelung des Ductus venosus Arantii _- fällt sehr früh, wiewohl dabei meist erst Hemiacardie und erst später Holoacardie eintritt. Die starke Ausbildung der Ersatznabelvenen weist auf langsamen, ja vielleicht oft sehr langsamen Eintritt der Holoacardie nach längerer Hemiacardic hin.

Acardie durch Nabelstenose wird meist erst ziemlich spät eintreten, solche dureh Nabelschnurbruch meist erheblich früher.

Acardie durch Verödung der einen Placentahälfte kann wohl im ganzen zweiten Viertel der Schwangerschaft zu Stande kommen. -

Wenn man also die Zeit des Eintrittes der Acardie ziemlich gut -- im einzelnen Fall manchmal sogar recht genau - - bestimmen kann, so ist dies doch nur in Rücksicht auf die Entstehungsart möglich, nicht oder wenigstens nicht annähernd eben so oft in Rücksicht auf die Form des Acardius. Denn diese hängt eben uur zum kleinen Theil von der Entstehungsart ab, zum grösseren von der Beschaffenheit der Anastomosen auf der Placenta, und diese hat zu der Zeit der Entstehung nur wenig Beziehungen. 
Es würde ermüden, wollte ich in dieser Richtung alle Combinationen durchsprechen. Wer sich darin eingehender unterrichten will, muss doch bezüglich der Entstehungsart die verschiedenen Gruppen und bezüglich der Form der Acardii die "anatomische Ausbildung" genauer studiren. -

Est ist nicht unmöglich, dass man in manchen f'ällen aucn. in der klinischen Beobachtung einen Anhalt gewinnt für die Zeit, in welcher die Acardie eingetreten ist.

Ich habe oben (Bd. 30, S. 365) einen Fall von Brummerstädt und dann (Bd. 55, S. 367) einen Fall von mir mitgetheilt, wo der Tod eines normalen oder wenigstens nicht herzlosen Zwillings bei fortdauernder Sohwangerschaft sowohl an der schnell eintretenden Verminderung der Beschwerden der Schwangeren, als auch durch das Centimetermaass deutlich zu erkennen und der Zeit nach ziemlich genau zu bestimmen war, indem das vordem so schnelle und beschwerliche Grössenwachsthum des Uterus plötzlich aufhörte oder sogar einer Verkleinerung Platz machte. Mit dem Tod des in Folge der Polyurie polyhydramnischen Zwillings hört dessen Polyurie auf und geht seine Polyhydramnie durch Resorption des Fruchtwassers ziemlich schnell zurück. Dies geschieht aber nicht allein bei Tod des polyhydramnischen Zwillings, sondern langsamer, wenn gleich auch bei Tod des oligohydramnischen, weil mit dessem Tod die Ursache des vermehrten arteriellen Druckes und der Herzhypertrophie und damit der Polyurie des polyhydramnischen Zwillings aufhört.

Aehnlich muss es auch bei Eintritt von Acardie sein. Einmal liefert der Acardius selbst vermöge seines geringen Blutdruckes nur noch sehr wenig Fruchtwasser. Dann aber schwindet, wie bei Gesammttod eines Zwillings, so auch bei Herztod desselben die Ursache des stark vergrösserten arteriellen Druckes für den normalen Zwilling. Polyurie und Polyhydramnie werden dadureh mindestens geringer. Freilich kann dies klinisch recht deutlich nur werden in den Fällen, wo die Acardie erst etwa im 4. und 5. Monat eintritt, so dass die Aenderung im Wachsthum des Uterus auffällig wird. 